TRANSACTIONS OF THE

AMERICAN MATHEMATICAL SOCIETY

Volume 353, Number 12, Pages 4729-4756

S 0002-9947(01)02849-5

Article electronically published on July 11, 2001

\title{
REPRESENTATION TYPE OF $q$-SCHUR ALGEBRAS
}

\author{
KARIN ERDMANN AND DANIEL K. NAKANO
}

\begin{abstract}
In this paper we classify the $q$-Schur algebras having finite, tame or wild representation type and also the ones which are semisimple.
\end{abstract}

\section{INTRODUCTION}

1.1. Let $A$ be a finite-dimensional algebra over a field $k$. Furthermore, let $\bmod (A)$ denote the category of finite-dimensional $A$-modules. The algebra $A$ has finite representation type if and only if there are only finitely many indecomposable modules in $\bmod (A)$. Otherwise, $A$ has infinite representation type. Algebras of infinite representation type can be divided into two mutually exclusive categories - those that have either tame representation type or wild representation type. An algebra $A$ has tame representation type if for each dimension $d$ there are finitely many 1-parameter families of modules which contain all $d$-dimensional indecomposable modules. In this situation there is a good chance one may be able to describe all indecomposable objects in $\bmod (A)$. On the other hand, algebras of wild representation type are those algebras whose representation theory is as complicated as modules over the free associative algebra $k\langle x, y\rangle$. Given a class of algebras a fundamental problem is to classify the representation type of the algebras and to gain as much insight into those of finite or tame type as possible.

Let $\mathrm{GL}_{n}(k)$ be the general linear group over $k$ and $V$ be the $n$-dimensional natural representation. The Schur algebra $S(n, d)$ is defined as $\operatorname{End}_{\Sigma_{d}}\left(V^{\otimes d}\right)$ where $\Sigma_{d}$ is the symmetric group on $d$ letters. These algebras arise naturally in the study of the polynomial representation theory of $\mathrm{GL}_{n}(k)$. In [Erd2], [DN], and DEMN], a complete classification of the representation type for the Schur algebra was obtained. The purpose of this paper is to provide a complete classification of the representation type of the quantum (or $q$ ) Schur algebras.

1.2. Definitions and Notation. Let $R$ be an integral domain and $q$ be a unit in $R$, we assume $q \neq 1$. Let us regard the symmetric group $\Sigma_{r}$ as the set of bijections of $\{1, \ldots, r\}$ where $r$ is a natural number which we assume to be $\geq 2$. The Hecke algebra $\mathcal{H}_{q}(r)=\mathcal{H}_{R, q}\left(\Sigma_{r}\right)$ is the free $R$-module with basis $\left\{T_{w}: w \in \Sigma_{r}\right\}$. The multiplication in the algebra is defined by the rule

$$
T_{w} T_{s}= \begin{cases}T_{w s} & \text { if } \ell(w s)>\ell(w), \\ q T_{w s}+(q-1) T_{w} & \text { otherwise }\end{cases}
$$

Received by the editors September 24, 1999 and, in revised form, September 13, 2000.

2000 Mathematics Subject Classification. Primary 16G60, $20 \mathrm{G} 42$.

Research of the second author partially supported by NSF grant DMS-9800960.

(C)2001 American Mathematical Society 
where $s=(i, i+1) \in \Sigma_{r}$ is a basic transposition and $w \in \Sigma_{r}$. The function $\ell: \Sigma_{r} \rightarrow \mathbb{N}$ is the usual length function.

For each partition $\lambda$ of $r$ there is a $q$-Specht module of the Hecke algebra $\mathcal{H}_{q}(r)$, denoted by $S^{\lambda}$. Assume that $R=k$ is a field and that $q \in k$ with $q \neq 0,1$.

Let $\lambda$ be a composition of $r$ and let $\Sigma^{\lambda}$ be the corresponding Young subgroup of $\Sigma_{d}$, so $\Sigma^{\lambda} \cong \Sigma_{\lambda_{1}} \times \Sigma_{\lambda_{2}} \times \cdots$. Let

$$
x_{\lambda}=\sum_{w \in \Sigma^{\lambda}} T_{w} .
$$

Define $M^{\lambda}:=x_{\lambda} \mathcal{H}_{q}(r)$. The module $M^{\lambda}$ has a unique submodule isomorphic to the Specht module $S^{\lambda}$, and there is a unique indecomposable direct summand of $M^{\lambda}$ containing $S^{\lambda}$; this is the Young module $Y^{\lambda}$. We have $Y^{\lambda} \cong Y^{\mu}$ if and only if $\lambda=\mu$. Each $M^{\alpha}$ (for $\alpha \in \Lambda(n, r)$ ) is a direct sum of such Young modules (see Mar).

The $q$-Schur algebra was introduced by Dipper and James as follows. Let $\Lambda(n, r)$ be the set of compositions of $r$ into $n$ parts. The $q$-Schur algebra is defined as

$$
S_{q}(n, r)=S_{R, q}(n, r)=\operatorname{End}_{\mathcal{H}_{q}(r)}\left(\bigoplus_{\lambda \in \Lambda(n, r)} x_{\lambda} \mathcal{H}_{q}(r)\right) .
$$

Note that if $q=1$ with $R=k$ where $k$ is a field, then this is the classical Schur algebra $S(n, r)=S_{k}(n, r) \mathrm{Gr}$.

The $q$-Schur algebra over a field can also be constructed via quantum $\mathrm{GL}_{n}$; here we use the version introduced in [DD], and we take advantage of the work done in Don. Let $A_{q}(n)$ be the bialgebra defined there; for each $r \geq 0$ its homogeneous part $A_{q}(n, r)$ is a finite-dimensional subcoalgebra, and its dual is therefore a finitedimensional algebra which can be identified with the $q$-Schur algebra. We note that the representation theory works well for arbitrary nonzero $q$.

1.3. Main results. For the remainder of the paper we will assume that $R=k$ is an algebraically closed field of characteristic $p \geq 0$ ( $p=0$ or a prime number). We make the convention that $p$ is arbitrary unless it is explicitly specified. We also assume $q \in k$ and $q \neq 0,1$ and $n \geq 2$. Let $l$ be the multiplicative order of $q$ in $k^{*}$, that is, either $q$ is a primitive $l$ th root of 1 , or $l=\infty$. Note that if $q$ is a primitive $l$ th root of 1 and $p>0$, then $l$ and $p$ must be coprime. The main results of this paper are listed below.

Theorem (A). The algebra $S_{q}(n, r)$ is semisimple if and only if one of the following holds:

(i) $q$ is not a root of unity;

(ii) $q$ is a primitive lth root of unity and $r<l$;

(iii) $n=2, p=0, l=2$ and $r$ is odd;

(iv) $n=2, p \geq 3, l=2$ and $r$ is odd with $r<2 p+1$.

Theorem (B). The algebra $S_{q}(n, r)$ has finite representation type but is not semisimple if and only if $q$ is a primitive lth root of unity with $l \leq r$, and one of the following holds:

(i) $n \geq 3$ and $r<2 l$;

(ii) $n=2, p \neq 0, l \geq 3$ and $r<l p$;

(iii) $n=2, p=0$ and either $l \geq 3$, or $l=2$ and $r$ is even; 
(iv) $n=2, p \geq 3, l=2$ and $r$ even with $r<2 p$, or $r$ is odd with $2 p+1 \leq r<$ $2 p^{2}+1$.

Theorem (C). The algebra $S_{q}(n, r)$ has tame representation type if and only if $q$ is a primitive lth root of unity and one of the following holds:

(i) $n=3, l=3, p \neq 2$ and $r=7,8$;

(ii) $n=3, l=2$ and $r=4,5$;

(iii) $n=4, l=2$ and $r=5$;

(iv) $n=2, l \geq 3, p=2$ or $p=3$ and $p l \leq r<(p+1) l$;

(v) $n=2, l=2, p=3$ and $r \in\{6,19,21,23\}$;

We remark that Theorem 1.3(A) corrects several misconceptions in the literature on the semisimplicity of quantum Schur algebras (see [PW] (11.4.1) Lem.], [Mar, p. 191 Rem. (1)], Don p. $96(7)]$ ). It is also interesting to note that when $p=0$ the algebra $S_{q}(2, r)$ is always semisimple, or of finite representation type.

1.4. For the sake of convenience it will be useful to reformulate the results given in the preceding section.

Theorem (A). The algebra $S_{q}(n, r)$ is not semi-simple if and only if $q$ is a primitive lth root of unity, such that $r \geq l$ and one of the following holds:

(a) $n \geq 3$;

(b) $n=2$ and $l \geq 3$;

(c) $n=2, l=2$ and $r$ even;

(d) $n=2, l=2, p \geq 3$ and $r$ odd with $r \geq 2 p+1$.

Theorem (B). The algebra $S_{q}(n, r)$ is of infinite type if and only if $q$ is a primitive lth root of unity and one of the following holds:

(a) $n \geq 3$ and $r \geq 2 l$;

(b) $n=2, p \neq 0, l \geq 3$ and $r \geq l p$;

(c) $n=2, p \geq 3, l=2$ and $r$ is even, $r \geq 2 p$, or $r$ is odd, $r \geq 2 p^{2}+1$.

Theorem (C). The algebra $S_{q}(n, r)$ has wild representation type if and only if $q$ is a primitive lth root of unity and one of the following holds:

(a) $n \geq 3, l \geq 4$ and $r \geq 2 l$;

(b) $n \geq 3, l=3$ and $r \geq 2 l$ except for the algebras in Theorem $1.3(C)(i)$;

(c) $n \geq 3, l=2$ and $r \geq 2 l$, except for the algebras in Theorem 1.3(C)(ii) and (iii);

(d) $n=2, l \geq 3, p \neq 0$ and $r \geq$ lp except for the algebras in Theorem 1.3(C)(iv);

(e) $n=2, l=2, p \geq 3$ and $r$ is odd, $r \geq 2 p^{2}+1$, or $r$ is even and $r \geq 2 p$, except for the algebras in Theorem 1.3 $(C)(v)$.

It may be surprising that, for example, when $n=3, l=3$ and $p \neq 2$ the algebra is wild for $r=6$ but tame when $r=7,8$. But algebras $S_{q}(n, r)$ and $S_{q}(n, r+t)$ for $1 \leq t<n$ and $r \geq n$ can be quite different; one can see this directly in small cases. Let $l=2$, then the algebra $S_{q}(2,2)$ is isomorphic to $k[T] /\left((T+1)^{2}\right)$ and hence is not semisimple. On the other hand, $S_{q}(2,3)$ is semisimple, there are just two simple modules and they lie in different blocks (and simple modules for $q$-Schur algebras have no non-split self-extensions).

We start with general reductions (in $\S 2$ ). In $\S 3$, we analyze the case when $n=2$. From these results we can present a complete proof of Theorems 1.3(A) and 1.4(A). In $\S 4$, we provide the proofs of Theorems $1.3(\mathrm{~B}), 1.3(\mathrm{C}), 1.4(\mathrm{~B})$ and $1.4(\mathrm{C})$, modulo 
studying the representation type of specific $q$-Schur algebras $S_{q}(n, d)$ for $n=2,3,4$. The remaining verifications will be accomplished in $\S \S 5$ and 6 .

Acknowledgements. The second author would like to acknowledge the Mathematical Institute at Oxford University for their support and hospitality during his visit in June 1997. We also thank the referee for many valuable suggestions.

\section{Preliminaries}

2.1. Let $A$ be a finite dimensional algebra. We say that $A$ has property $\left({ }^{*}\right)$ if $A$ has one of the following properties:

- $A$ is not semisimple,

- $A$ has infinite representation type,

- $A$ has wild representation type.

2.2. Let $A$ and $B$ be two finite-dimensional algebras. Let $F$ be an additive functor from $\bmod (A)$ to $\bmod (B)$ which preserves indecomposables and reflects isomorphisms. If such a functor exists then it is easy to see that (i) if $A$ is not semisimple, then $B$ is not semisimple, provided $F$ is also either left exact or right exact, (ii) if $A$ has infinite representation type, then $B$ has infinite representation type, and (iii) if $A$ is wild, then so is $B$ (this follows directly from the characterization via a bimodule). In many situations such a functor can be found.

2.3. The following two propositions give sufficient conditions for recognizing an algebra of wild representation type.

Proposition (A). Let $A$ be a finite-dimensional algebra with primitive orthogonal idempotents $e_{1}$ and $e_{2}$. Let $P=e_{1} A$ and $e$ be the idempotent $e=e_{1}+e_{2}$. Suppose $e P$ has radical quotient $\operatorname{rad}_{e A e}(e P) / \operatorname{rad}_{e A e}^{2}(e P)=S_{1} \oplus S_{1} \oplus S_{2}$ where $S_{i}$ is simple corresponding to $e_{i}$. Then $A$ is wild.

Proof. It suffices to show that $e A e / \operatorname{rad}_{e A e}^{2}(e A e)$ is wild. Consider the composition of the following functors: first, the natural functor from $\bmod \left(e A e / \operatorname{rad}^{2}(e A e)\right)$ to $\bmod (A)$, then the functor $-\otimes_{e A e} e A$. Clearly the first functor has the same properties as in 2.2, and the second one as well which can be seen by considering its right adjoint. By the hypothesis, the quiver for this algebra contains a connected quiver with two vertices and two loops at one of the vertices (see Figure 1). Any such algebra is wild by comparison with the connected quiver with three vertices and three arrows whose dual is listed in [Ri1].

Proposition (B). Let $A$ be a finite-dimensional algebra such that $\operatorname{Ext}_{A}^{1}(S, T) \cong$ $\operatorname{Ext}_{A}^{1}(T, S)$ for any two simple modules $S, T$ of $A$. Suppose the quiver of $A$ contains a proper subquiver which is a circuit with at least 3 vertices. Then $A$ is wild.

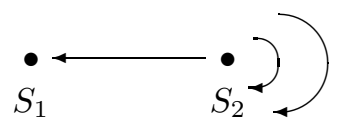

FiguRE 1. 
Proof. The separated quiver for $A$ contains $\tilde{A_{n}}$ which arises from this circuit. This is a proper subquiver and the condition on the extensions implies that the separated quiver contains $\tilde{A}_{n}$ extended by one arrow. Therefore, $A$ is wild. One can see this by exhibiting a functor from the category of representations of $\widetilde{\widetilde{E}}_{7}$ into the category of $A$-modules which preserves indecomposability and reflects isomorphisms.

2.4. We will now focus our attention on $q$-Schur algebras. The results in this subsection will be used to reduce the general question of representation type of $q$-Schur algebras to looking at the representation type of $S_{q}(n, r)$ for $n=2,3,4,5$ and small $r$. Our first result compares the representation type of $S_{q}(n, r)$ with the representation type of $S_{q}\left(n^{\prime}, r\right)$ where $n^{\prime} \geq n$.

Proposition (A). If $S_{q}(n, r)$ has property $(*)$, then $S_{q}\left(n^{\prime}, r\right)$ has property $\left({ }^{*}\right)$ for $n^{\prime} \geq n$.

Proof. There exists an idempotent $e$ in $S_{q}\left(n^{\prime}, r\right)$ such that $e S_{q}\left(n^{\prime}, r\right) e \cong S_{q}(n, r)$ Don 2.2(1) p. 48]. Set $A=S_{q}\left(n^{\prime}, r\right)$ and $e A e=S_{q}(n, r)$. Let $F$ be the functor defined from $\bmod (e A e)$ to $\bmod (A)$ by $F(-)=A e \otimes_{e A e}(-)$. This functor preserves indecomposables and reflects isomorphisms, and is right exact. Furthermore, from Erd1 I 4.7], if $e A e$ has wild representation type, then so does $A$.

Let $M$ be a (right) $S_{q}(n, r)$-module, $N$ a $k\langle x, y\rangle$-module, and $Q$ an $S\left(n, r^{\prime}\right)-$ $k\langle x, y\rangle$-bimodule which is free as right $k\langle x, y\rangle$-module. With these assumptions $M \otimes_{k} Q$ becomes an $S_{q}\left(n, r+r^{\prime}\right)-k\langle x, y\rangle$-bimodule. The left action on this tensor product is given by the bialgebra structure on quantum matrix space. On the other hand, the right actions of $k\langle x, y\rangle$ are given by $(m \otimes q) \cdot a=m \otimes(q . a)$ for $m \in M$, $q \in Q$ and $a \in k\langle x, y\rangle$. Moreover, there exists an isomorphism of $S_{q}\left(n, r_{1}+r_{2}\right)$ modules:

$$
M \otimes_{k}\left[Q \otimes_{k\langle x, y\rangle} N\right] \cong\left[M \otimes_{k} Q\right] \otimes_{k\langle x, y\rangle} N .
$$

Let $\delta$ be the one-dimensional determinant representation of quantum $\mathrm{GL}_{n}$ which also may be regarded as a representation of $S(n, n)$. We will use this representation to relate the representation type of $S_{q}(n, r)$ with $S_{q}(n, r+n)$.

Proposition (B). If $S_{q}(n, r)$ has property $(*)$, then $S_{q}(n, n+r)$ has property $\left(^{*}\right)$.

Proof. The statement of the proposition concerning semisimplicity and infinite representation type will hold if there exists an exact functor from $\bmod \left(S_{q}(n, r)\right)$ to $\bmod \left(S_{q}(n, n+r)\right)$ which preserves indecomposability and reflects isomorphisms. The functor defined by $G(-)=\delta \otimes(-)$ has the required properties.

Now suppose that $S_{q}(n, r)$ has wild representation type. Then there exists a $S_{q}(n, r)-k\langle x, y\rangle$-bimodule $Q$ such that the functor $F(-)=Q \otimes_{k\langle x, y\rangle}-$ preserves indecomposability and reflects isomorphisms. Consider the functor $F^{\prime}$ from $\bmod (k\langle x, y\rangle)$ to $\bmod \left(S_{q}(n, r+n)\right)$ defined by $F^{\prime}(-)=\left[\delta \otimes_{k} Q\right] \otimes_{k\langle x, y\rangle}-$.

We will first show that $F^{\prime}$ preserves indecomposability. Assume that $N$ is an indecomposable $k\langle x, y\rangle$-module and $F^{\prime}(N) \cong X_{1} \oplus X_{2}$. Working in the category of rational representations, we have

$$
\begin{aligned}
\left(\delta^{-1} \otimes X_{1}\right) \oplus\left(\delta^{-1} \otimes X_{2}\right) & \cong \delta^{-1} \otimes_{k} F^{\prime}(N) \\
& \cong \delta^{-1} \otimes_{k}\left[\left[\delta \otimes_{k} Q\right] \otimes_{k\langle x, y\rangle} N\right] \\
& \cong Q \otimes_{k\langle x, y\rangle} N \\
& \cong F(N) .
\end{aligned}
$$


Therefore, since $F$ preserves indecomposability, $F^{\prime}$ must also preserve indecomposability.

Next we will prove that $F^{\prime}$ reflects isomorphisms. Let $F^{\prime}\left(N_{1}\right) \cong F^{\prime}\left(N_{2}\right)$. By tensoring both sides of this isomorphism by $\delta^{-1}$ we have $Q \otimes_{k\langle x, y\rangle} N_{1} \cong Q \otimes_{k\langle x, y\rangle} N_{2}$. The functor $F$ preserves isomorphisms so $N_{1} \cong N_{2}$. Hence, $S_{q}(n, r+n)$ has wild representation type.

Let $\mathrm{GL}_{n, q}$ denote quantum $\mathrm{GL}_{n}$. Moreover, let $X(T)$ be the weight lattice and let $X(T)_{+}$be the set of dominant integral weights. For any $\mathrm{GL}_{n, q}$-module $V$ and $\lambda, \mu \in X(T)$, define $p r_{\lambda} V$ to be the largest $\mathrm{GL}_{n, q}$-submodule of $V$ such that $\mu \in$ $W_{p} \cdot \lambda$ for every composition factor $L_{q}(\mu)$ of $V$. Let $\Phi^{+}$be a set of positive roots for the root system of type $A_{n-1}$, and let $\rho$ be the half-sum of positive roots and

$$
\bar{C}_{\mathbb{Z}}=\left\{\lambda \in X(T) \mid 0 \leq\left\langle\lambda+\rho, \alpha^{\vee}\right\rangle \leq p \text { for all } \alpha \in \Phi^{+}\right\} .
$$

Moreover, let $\lambda, \mu \in \bar{C}_{\mathbb{Z}}$ and let $\nu$ be the unique weight in $X(T)_{+} \cap W(\mu-\lambda)$. The translation functor $T_{\lambda}^{\mu}: \bmod \left(\mathrm{GL}_{n, q}\right) \rightarrow \bmod \left(\mathrm{GL}_{n, q}\right)$ is defined by

$$
T_{\lambda}^{\mu}(V)=p r_{\mu}\left(L_{q}(\nu) \otimes p r_{\lambda} V\right) .
$$

Let $\mathcal{P}_{q}(n)$ be the category of polynomial modules of $\mathrm{GL}_{n, q}$ and let $\mathcal{F}$ be the functor from $\bmod \left(\mathrm{GL}_{n, q}\right)$ to $\mathcal{P}_{q}(n)$ defined by taking the largest $\mathcal{P}_{q}(n)$-submodule of a given $\mathrm{GL}_{n, q}$-module. For any $\lambda, \nu \in \bar{C}_{\mathbb{Z}}$, define a truncated translation functor $\hat{T}_{\lambda}^{\mu}$ to be the composite $\mathcal{F} \circ T_{\lambda}^{\mu}$. By restriction $\hat{T}_{\lambda}^{\mu}$ induces a functor from $\mathcal{P}_{q}(n)$ into itself.

Proposition $(\mathbf{C})$. Let $\mathcal{B}(\lambda)$ be a block of $S_{q}(n, r)$ containing the simple module $L_{q}(\lambda)$. Suppose that $\mu \in \Lambda^{+}\left(n, r^{\prime}\right)$ is a weight in the same facet as $\lambda$ with $\mu-\lambda \in$ $P(D)$. If $\mathcal{B}(\lambda)$ has property $(*)$ then $S_{q}\left(n, r^{\prime}\right)$ has property $\left(^{*}\right)$.

For the definition of "facet", we refer to [Jan, p. 261], and $P(D)$ is the set of polynomial weights, which we identify with $\mathbb{N}^{n}$.

Proof. Under the hypotheses given in the theorem, $\hat{T}_{\mu}^{\lambda} \circ \hat{T}_{\lambda}^{\mu}$ is equivalent to the identity functor. This shows that the functor $\hat{T}_{\lambda}^{\mu}$ preserves indecomposable modules and isomorphism classes. Therefore, the statement of this proposition concerning semisimplicity and infinite representation type holds.

Suppose that $\mathcal{B}(\lambda)$ has wild representation type. Let $F$ be the functor from $\bmod (k\langle x, y\rangle)$ to $\bmod (\mathcal{B}(\lambda))$ defined by $F(-)=Q \otimes_{k\langle x, y\rangle}-$ where $Q$ is a $\mathcal{B}(\lambda)-$ $k\langle x, y\rangle$-bimodule which is free as a right $k\langle x, y\rangle$-module. Furthermore, let $F^{\prime}$ be the functor from $\bmod (k\langle x, y\rangle)$ to $\bmod (\mathcal{B}(\mu))$ defined by $F^{\prime}(-)=\hat{T}_{\lambda}^{\mu}(Q) \otimes_{k\langle x, y\rangle}-$. We have $\hat{T}_{\lambda}^{\mu}(Q)$ is a $\mathcal{B}(\mu)-k\langle x, y\rangle$-bimodule which is also free as a $k\langle x, y\rangle$-module, also a direct verification shows that $F^{\prime}=\hat{T}_{\lambda}^{\mu} \circ F$.

We will first show that $F^{\prime}$ takes indecomposables to indecomposables. Let $N$ be an indecomposable $k\langle x, y\rangle$-module. Let $F^{\prime}(N)=\hat{T}_{\lambda}^{\mu} \circ F(N) \cong X_{1} \oplus X_{2}$ where $X_{j} \neq\{0\}$ for $j=1,2$. Since $\hat{T}_{\mu}^{\lambda} \circ \hat{T}_{\lambda}^{\mu}$ is equivalent to the identity functor, we have $\hat{T}_{\mu}^{\lambda}\left(X_{1}\right) \oplus \hat{T}_{\mu}^{\lambda}\left(X_{2}\right) \cong F(N)$. Observe that $\hat{T}_{\mu}^{\lambda}\left(X_{j}\right) \neq\{0\}$ for $j=1,2$. Since $X_{j} \neq 0$, there exist simple modules $L_{q}\left(\gamma_{j}\right)$ with $\gamma_{j} \in W_{p} \cdot \mu$ which are submodules of $X_{j}$ for $j=1,2$. Therefore, $0 \neq \hat{T}_{\mu}^{\lambda}\left(L_{q}\left(\gamma_{j}\right)\right) \hookrightarrow \hat{T}_{\mu}^{\lambda}\left(X_{j}\right)$ for $j=1$, 2 . This contradicts the fact that $F$ preserves indecomposability, thus $F^{\prime}$ must preserve indecomposability. 
Finally, we show that $F^{\prime}$ preserves isomorphism classes. Assume that $F^{\prime}\left(N_{1}\right) \cong$ $F^{\prime}\left(N_{2}\right)$. Therefore,

$$
F\left(N_{1}\right) \cong \hat{T}_{\mu}^{\lambda}\left(\hat{T}_{\lambda}^{\mu} F\left(N_{1}\right)\right) \cong \hat{T}_{\mu}^{\lambda}\left(\hat{T}_{\lambda}^{\mu} F\left(N_{2}\right)\right) \cong F\left(N_{2}\right)
$$

and $N_{1} \cong N_{2}$, and $F^{\prime}$ preserves isomorphism classes. Hence, $\mathcal{B}(\mu)$ must have wild representation type.

2.5. The $q$-Schur algebras are quasi-hereditary algebras [PW, 11.5], [Mar, Thm. 7.5.1]. In examining the representation theory of the $q$-Schur algebras we will extensively make use of the representation theory of quasi-hereditary algebras [Ri2], Don Appendix]. A quick review of this subject is presented below.

Let $A$ be a finite-dimensional algebra $A$ with simple modules $L(\lambda)$ labelled by $\lambda \in$ $\Lambda$ where $(\Lambda, \leq)$ is a partially ordered set. Let $P(\lambda)$ be the projective cover of $L(\lambda)$. The standard module $\Delta(\lambda)$ is defined to be the largest quotient of the projective $P(\lambda)$ which has only composition factors $L(\mu)$ with $\mu \leq \lambda$. The costandard module $\nabla(\lambda)$ is defined similarly, as a submodule of the injective hull $I(\lambda)$ of $L(\lambda)$. The algebra $A$ is quasi-hereditary if for each $\lambda \in \Lambda$ :

(2.5.1) $L(\lambda)$ occurs only once as a composition factor of $\Delta(\lambda)$;

(2.5.2) $P(\lambda)$ has a filtration with sections isomorphic to standard modules where $\Delta(\lambda)$ occurs only once, and if $\Delta(\mu)$ occurs, then $\mu \geq \lambda$.

Quasi-hereditary algebras satisfy "Brauer-Humphreys reciprocity". Let $[P(\lambda)$ : $\Delta(\mu)$ ] denote the number of times $\Delta(\mu)$ appears as a section in a $\Delta$-filtration of $P(\lambda)$.

(2.5.3) Then $[P(\lambda): \Delta(\mu)]=[\Delta(\mu): L(\lambda)]$.

Let $\mathcal{F}(\Delta)$ be the category of all $A$-modules which have a $\Delta$-filtration and similarly $\mathcal{F}(\nabla)$ be the category of all $A$-modules which have a $\nabla$-filtration. Then for each $\lambda \in$ $\Lambda$ there is a unique indecomposable module denoted by $T(\lambda)$ with highest weight $\lambda$ which has both a $\Delta$-filtration and also a $\nabla$-filtration [Ri2], Don. Moreover, these are all indecomposable modules which belong to $\mathcal{F}(\Delta) \cap \mathcal{F}(\nabla)$. We call any module which is a direct sum of such $T(\lambda)$ a tilting module.

Let $T:=\bigoplus_{\lambda} T(\lambda)^{n(\lambda)}$ with $n(\lambda) \geq 1$ for all $\lambda$. Consider the algebra $A^{\prime}:=$ End $_{A}(T)$, which is again quasi-hereditary. This algebra is known as the Ringel dual of $A$, and is unique up to Morita equivalence. Furthermore, $\left(A^{\prime}\right)^{\prime}$ is Morita equivalent to $A$. According to Ri2, the functor $F=\operatorname{Hom}_{A}(T,-)$ induces an equivalence $\mathcal{F}_{A}(\nabla) \rightarrow \mathcal{F}_{A^{\prime}}(\Delta)$, taking $\nabla(\lambda)$ to $\Delta^{\prime}(\lambda)$ and $T(\lambda)$ to the projective $P^{\prime}(\lambda)$. It follows that

$(2.5 .4)[T(\lambda): \nabla(\mu)]=\left[P^{\prime}(\lambda): \Delta^{\prime}(\mu)\right]=\left[\Delta^{\prime}(\mu): L^{\prime}(\lambda)\right]$,

where the last step uses the reciprocity law for $A^{\prime}$. Since $\left(A^{\prime}\right)^{\prime}$ is Morita equivalent to $A$ with standard modules identified, it also follows that

(2.5.5) $\left[T^{\prime}(\lambda): \nabla^{\prime}(\mu)\right]=[P(\lambda): \Delta(\mu)]=[\Delta(\mu): L(\lambda)]$.

If $A$ is quasi-hereditary, then $\operatorname{Ext}_{A}^{1}(\Delta(\gamma), \Delta(\mu)) \neq 0$ implies $\gamma<\mu$. Moreover, for $\mu \nsupseteq \lambda$

$$
\operatorname{Hom}_{A}\left(\operatorname{rad}_{A} \Delta(\lambda), L(\mu)\right) \cong \operatorname{Ext}_{A}^{1}(L(\lambda), L(\mu)) .
$$

In particular, $\operatorname{Ext}_{A}^{1}(L(\lambda), L(\lambda))=0$.

Suppose that $A$ has a duality $(-)^{0}$ fixing the simple modules. For the $q$-Schur algebra this is given by the transposed dual. In this case $\Delta(\lambda) \cong(\nabla(\lambda))^{0}$ and also $T(\lambda)^{0} \cong T(\lambda)$. Therefore, $[T(\lambda): \nabla(\mu)]=[T(\lambda): \Delta(\mu)]$. Furthermore, if $A$ is a quasi-hereditary algebra with duality, then for any $\lambda, \mu$ we have $\operatorname{Ext}_{A}^{1}(L(\lambda), L(\mu)) \cong$ $\operatorname{Ext}_{A}^{1}(L(\mu), L(\lambda))$. Since $\lambda \neq \mu$ implies either $\mu \nsupseteq \lambda$ or $\lambda \nsupseteq \mu$, we deduce that the 
quiver of $A$ can be obtained by knowing the second radical layer of the standard modules.

By considering the Ringel duality from $A^{\prime}$ to $A$ one sees that if $T_{A^{\prime}}(\lambda)$ is injective, then the $A$-module $P(\lambda)$ must be a tilting module.

2.6. The reduction theorems in Section 2.4 will allow us to reduce questions of representation type to $S_{q}(n, r)$ for $n=2,3,4,5$, and $r$ small. With these reductions we will make explicit computations of the quiver and relations for these algebras. This will be accomplished in the following way. In order to study the quiver and relations for the algebra $S_{q}(n, r)$, it is enough to consider its basic algebra, that is, the algebra

$$
\operatorname{End}_{S_{q}(n, r)}\left(\bigoplus_{\lambda \in \Lambda^{+}(n, r)} P(\lambda)\right)
$$

where the set indexing the simples is $\Lambda^{+}(n, r)$. Because we are looking at small rank cases it will be possible to determine the structure of the projective modules $P(\lambda)$ for $q$-Schur algebras. From this information one can then compute out the quiver and relations for this algebra. This technique was used effectively in [DEMN] for this purpose. In some instances we will refer to that paper for the detailed computations.

The standard modules are the usual $q$-Weyl modules which we denote by $\Delta(\lambda)$. There is a duality fixing the simple modules; hence the quiver of a $q$-Schur algebra can be obtained from knowing the structures of the $q$-Weyl modules. Often it will be enough for us to find the quiver. The $q$-Weyl modules we study will mostly be duals of $q$-symmetric powers. The submodule structure of these is completely understood by [Th, 3.4, 3.9]; we will use this frequently without further comment. In general, the dimension of $\Delta(\lambda)$ is given by Weyl's character formula.

Another method for computing the quiver and relations for $q$-Schur algebras is to compute homomorphisms between Young modules. From Section 1.2 the algebra $\operatorname{End}\left(\bigoplus_{\lambda \in \Lambda^{+}(n, r)} Y^{\lambda}\right)$ is a basic algebra for $S_{q}(n, r)$. If $\mathcal{B}$ is a block of the Hecke algebra $\mathcal{H}_{q}(r)$, then let $S_{\mathcal{B}}$ be the endomorphism ring of $\bigoplus_{D^{\lambda} \in \mathcal{B}} Y^{\lambda}$. The algebra $S_{\mathcal{B}}$ is the basic algebra for a sum of blocks of $S_{q}(n, r)$ (usually just one block). As in Erd2, for certain parameters $r$ and $\mathcal{B}$, one determines the submodule structure of the $Y^{\lambda}$ and by applying results from [Erd2] a presentation for $S_{\mathcal{B}}$ by quivers and relations is obtained. We recall that the blocks of the Hecke algebra are described by the 'Nakayama conjecture' (see [JM]).

The structure of the $Y^{\lambda}$ is to some extent also found via the $q$-Schur algebra (the method in [Erd2] was different). The Young modules have a Specht filtration, and to understand their structure we first need to know the filtration multiplicities and second the decomposition numbers $\left[S^{\mu}: D^{\lambda}\right]$ for the Hecke algebra.

The Young module $Y^{\lambda}$ is projective if and only if $\lambda$ is $l$-restricted. However, the reciprocity formula $\left[Y^{\lambda}: S^{\mu}\right]=[\Delta(\mu): L(\lambda)]$ holds for arbitrary $\lambda$ : There is a quotient of the Hecke algebra for which the modules $S^{\lambda}$ and $Y^{\lambda}$ for 2-part partitions $\lambda$ survive, which is isomorphic to a Ringel dual of $S_{q}(2, r)$ (almost, if $l=2$ ) in such a way that the Young modules are identified with the tilting modules and the Specht modules with the standard modules. (cf. [Don, 4.7]). Therefore this identity holds by (2.5.4). Similarly, if $\lambda$ is $l$-regular, then

$$
\left[S^{\mu}: D^{\lambda}\right]=[T(\lambda): \Delta(\mu)] .
$$




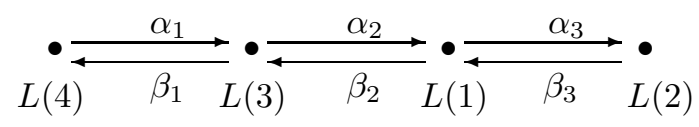

Figure 2.

The filtration multiplicities of $T(\lambda)$ can be studied from its structure as twisted tensor products; and for $n=2$ this gives a complete answer (Don, 3.3, 3.4], see also §3.1). As a further tool we use row- and column-removal, from ([Don, 4.2(9), $(15)])$.

If $Y^{\lambda}$ is projective and if all partitions in the block of $\lambda$ are $l$-regular, the projective $P(\lambda)$ for the Schur algebra is a tilting module.

2.7. In DEMN, 3.5], a basic algebra was defined by quiver and relations, which will be denoted by $\mathcal{S}$. The quiver for $\mathcal{S}$ is given in Figure 2, with the relations $\alpha_{1} \beta_{1}=\alpha_{2} \beta_{2}=\beta_{3} \alpha_{3}=0, \alpha_{1} \alpha_{2} \alpha_{3}=0=\beta_{3} \beta_{2} \beta_{1}, \beta_{1} \alpha_{1} \alpha_{2}=\alpha_{2} \alpha_{3} \beta_{3}$, and $\beta_{2} \beta_{1} \alpha_{1}=$ $\alpha_{3} \beta_{3} \beta_{2}$. When the characteristic of the field is 2, then the classical Schur algebra $S(2,6)$ is Morita equivalent to $\mathcal{S}$, and it was shown to have wild representation type. This algebra will arise in several different places so it would be nice to be able to identify $\mathcal{S}$ by simply knowing the decomposition matrix and some conditions on the projectives.

Proposition. Let $A$ be a quasi-hereditary algebra with duality fixing the simple modules. Moreover, assume that $A$ has four simple modules $L(i)$ where $i \in\{1,2$, $3,4\}$ and $<$ is the natural order. Suppose $A$ has decomposition matrix of the form

$$
\left(\begin{array}{l}
{[\Delta(4): L(\mu)]} \\
{[\Delta(3): L(\mu)]} \\
{[\Delta(2): L(\mu)]} \\
{[\Delta(1): L(\mu)]}
\end{array}\right)=\left(\begin{array}{llll}
1 & 1 & 0 & 1 \\
0 & 1 & 1 & 1 \\
0 & 0 & 1 & 1 \\
0 & 0 & 0 & 1
\end{array}\right)
$$

where the rows and columns are indexed by decreasing weights. Also, assume that $P(1)$ and $P(2)$ are injective. Then $A$ is Morita equivalent to $\mathcal{S}$. Furthermore, $\mathcal{S}$ has wild representation type.

Proof. Injective modules for quasi-hereditary algebras have $\nabla$-filtrations. Therefore, if $P(1)$ is injective, then it is a tilting module and self-dual, so it is isomorphic to $I(1)$. By reciprocity, the filtration sections of $P(1)$ can be seen from the last column of the matrix. Using Ext-properties for quasi-hereditary algebras, it follows that $P(1)$ has a filtration

$$
P(1)=M_{0} \supset M_{1} \supset \cdots \supset M_{4}=0
$$

such that $M_{i-1} / M_{i} \cong \Delta(i)$. So $L(4)$ is the composition factor with the highest weight in $P(1)$ hence $P(1) \cong T(4)$. Moreover, we have that $\Delta(4)$ is a submodule of $P(1) \cong I(1)$, so the socle of $\Delta(4)$ is simple and isomorphic to $L(1)$. It follows that $\Delta(4)$ is uniserial, with heart $L(3)$.

We claim that $M_{2} \cong P(3)$. The module $M_{2}$ is indecomposable since its socle is simple, and it has the same $\Delta$-quotients as $P(3)$, so it suffices to show that $\operatorname{Ext}_{A}^{1}(\Delta(3), \Delta(4)) \cong k$. This is clear once one applies $\operatorname{Hom}_{A}(-, \Delta(4))$ to the exact sequence

$$
0 \rightarrow \Delta(4) \rightarrow P(3) \rightarrow \Delta(3) \rightarrow 0 .
$$


In particular, it follows that $P(3)$ has a simple socle $L(1)$.

Since $P(2)$ is also injective, it follows that $\Delta(3)$ has socle $L(2)$ and is therefore uniserial, and $P(2)$ must be uniserial with composition factors

$$
L(2), L(1), L(3), L(1), L(2) .
$$

With this information it is straightforward to verify the relations. The algebra $\mathcal{S}$ is wild because $P(1)$ has a quotient $T(1)$ which is uniserial with composition factors $L(1), L(2), L(1)$ and it also has a uniserial quotient with composition factors $L(1), L(3), L(1)$. It follows that $\operatorname{End}_{A}(P(1))$ is a Kronecker algebra. Now apply the Proposition 2.3(A) with $e_{1}$ and $e_{4}$.

\section{Representation type FOR $S_{q}(2, r)$}

3.1. We will first review some facts on $q$-Weyl modules and tilting modules for quantum $\mathrm{GL}_{2}$, from [Don] and [Cox2]. Let $q$ be an $l$ th root of unity where $l>1$ and let $k$ be a field of characteristic $p$ or of characteristic zero. Moreover, let $F: \mathrm{GL}_{n, q} \rightarrow \mathrm{GL}_{n}$ be the Frobenius map. The following notation will be used for weights [Cox2, 3.3] when $n=2$. For a restricted partition $\lambda=\left(\lambda_{1}, \lambda_{2}\right)$ we set $\delta=\lambda_{2}$ and $\mu=\lambda_{1}-\lambda_{2}$. Thus

$$
\lambda=(\mu+\delta, \delta)
$$

where $0 \leq \mu \leq l-1$ and $\delta \geq 0$. Now we consider weights satisfying $0 \leq \mu \leq l-2$. Define $\bar{\mu}$ by $\mu+\bar{\mu}=l-2$. Next we define

$$
\tilde{\lambda}:=\left(\lambda_{2}-1, \lambda_{1}+1-l\right)=\bar{\mu} \rho+(\mu-l+1+\delta) \varpi
$$

where $\rho=(1,0)$ and $\varpi=(1,1)$.

First consider the Weyl modules. We have for $n \geq 1$

$$
\Delta(l n-1+\delta, \delta) \cong \Delta(l-1+\delta, \delta) \otimes \Delta((n-1) \rho)^{F} .
$$

Moreover, there is a non-split exact sequence of $G$-modules

$$
\begin{aligned}
0 & \rightarrow \Delta(n-1) \rho)^{F} \otimes \Delta(\tilde{\lambda}+l \varpi) \rightarrow \Delta(\lambda+\ln \rho) \\
& \rightarrow \Delta(n \rho)^{F} \otimes \Delta(\lambda) \rightarrow 0
\end{aligned}
$$

Since here Weyl modules for restricted highest weights are simple, (3.1.3) and (3.1.4) determine completely the composition factors for Weyl modules.

Now let us consider the tilting modules. If $\lambda$ is restricted, then $\Delta(\lambda)$ is simple and hence isomorphic to $T(\lambda)$. The tilting module $T(\tilde{\lambda}+l \rho)$ has two $\Delta$-quotients, namely $\Delta(\tilde{\lambda}+l \rho)$ and $\Delta(\lambda)$. If $\tau=(\tilde{\lambda}+l \rho)+l \alpha$ (where $\alpha$ is an arbitrary partition), then we have

$$
T(\tau) \cong T(\tilde{\lambda}+l \rho) \otimes T(\alpha)^{F} .
$$

This allows one to work inductively since $T(\tilde{\lambda}+l \rho) \otimes \Delta(n \rho)^{F}$ has a $\Delta$-filtration; there is an exact sequence

$$
\begin{aligned}
0 & \rightarrow \Delta(\tilde{\lambda}+(n+1) l \rho) \rightarrow T(\tilde{\lambda}+l \rho) \otimes \Delta(n \rho)^{F} \\
& \rightarrow \Delta(\lambda+\ln \rho) \rightarrow 0 .
\end{aligned}
$$


3.2. Let $S=S_{q}(2, r)$ where $q$ is a primitive $l$ th root of unity. Every block component $S_{\mathcal{B}}$ is Morita equivalent to the block component of a principal block of some $q$-Schur algebra $S_{q}(n, d)$ for some $d \leq r$. To see this, apply the method in $\operatorname{Erd2}$ 1.3] (for the $q$-analogue, see [Don, 4.2(18)]). If $\mathcal{B}$ is not principal, then this induces an isomorphism of $S_{\mathcal{B}}$ with $S_{q}(2, r-2)_{\tilde{\mathcal{B}}}$ where $\tilde{\mathcal{B}}$ is the block whose core is obtained from that of $\mathcal{B}$ by removing the first column. If the new core has only one part, then $\tilde{\mathcal{B}}$ is principal; otherwise repeat the process.

Let $r=w l+l-1$. Then the functor $X \rightarrow X^{F} \otimes S t$ from the category of modules for the classical Schur algebra $S(n, w)$ to the category of $S_{q}(n, r)$-modules gives an equivalence of $S(n, w)$-mod with the principal block component of $S_{q}(n, r)$, this is the $q$-analogue of [Jan, II.10.5]; a variation of [Cox1, 5.4].

Lemma. The principal block component of $S_{q}(2, w l+l-1)$ is Morita equivalent to the classical Schur algebra $S(2, w)$, over the same field.

3.3. We consider the principal block component of $S_{q}(2, r)$ for small $r$ in detail. Let $r=w l+t$ where $w \geq 0$ and $0 \leq t \leq l-1$. Let $\mathcal{B}$ be the block of $\mathcal{H}_{q}(r)$ with core $(t)$. In the case when $t<l-1$, the component $S_{\mathcal{B}}$ contains $w+1$ simple modules, labelled by the following 2-part partitions:

$$
\begin{aligned}
\lambda(s)=(r-s l, s l) \quad \text { for } 0 \leq s \leq w / 2 & \text { or }(w-1) / 2, \\
\mu(s):=(r-s l-t-1, s l+t+1) & \\
\text { for } 0 \leq s \leq w / 2-1 & \text { or }(w-1) / 2 .
\end{aligned}
$$

Denote by $J_{m}$ the $m \times m$ Jordan block matrix which is an upper triangular matrix with ones along the diagonal and superdiagonal.

Proposition (A). Let $r=w l+t$ where $0 \leq t \leq l-1$, and $0<w$. If $k$ has characteristic $p$, then also assume that $w \leq p-1$.

(a) If $t=l-1$, then $S_{\mathcal{B}}$ is semisimple.

(b) Assume $t \neq l-1$. Then $S_{\mathcal{B}}$ is a block of $S_{q}(2, r)$ which is Morita equivalent to the algebra $\mathcal{A}_{w+1}$.

We recall from [Erd2] the definition of $\mathcal{A}_{m}$. It has quiver given in Figure 3 and is defined by the following relations:

$$
\begin{gathered}
\alpha_{i} \alpha_{i+1}=0, \quad \beta_{i+1} \beta_{i}=0, \quad \alpha_{1} \beta_{1}=0, \\
\beta_{i} \alpha_{i}=\alpha_{i+1} \beta_{i+1}, \quad(1 \leq i \leq m-2) .
\end{gathered}
$$

Proof. Part (a) holds by Lemma 3.2 since $w \leq p-1$ so that $S(2, w)$ is semisimple.

(b) Assume $r=w l+t$ where $0 \leq t<l-1$. We consider first the decomposition matrix for the Specht modules of the Hecke algebra $\mathcal{H}_{q}(r)$ of the partitions listed in (3.3.1) above. This has the form $\left(J_{w+1}\right)^{t}$, for $w \leq p$, with the exception that if

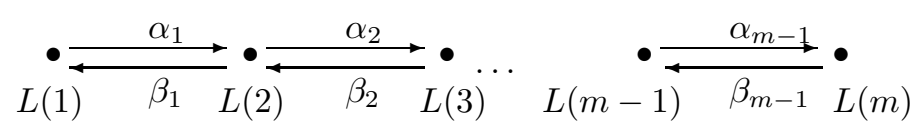

FiguRE 3. 
$l=2$ and the degree is even, then the last column has to be removed. This can be proved by using the tilting modules (see $\S 2.6, \S 3.1$ and [Don, 4.4(6)]).

The filtration multiplicities of the Young modules are equal to decomposition numbers for Weyl modules; see $\S 2.6$. We find that for $w<p$, this matrix has form $\left(J_{w+1}\right)^{t}$. For $w=p$ it is almost $\left(J_{w+1}\right)^{t}$ except that the first row is $[1,1,1,0, \cdots 0]$. This comes from the fact that the module $\Delta(l p)$ has length three; and the $\mathrm{GL}_{2}(k)$ module $\Delta(p)$ is not simple anymore.

Note that Young modules are self-dual. Therefore it follows easily from the above mulitplicities, that the Young modules for the Hecke algebra have the structure of the modules in Erd2, (3.2)]. The statement of the result now follows from [Erd2 $(3.2)]$.

Proposition (B). Assume that $k$ has characteristic $p \geq 2$. Let $r=p l+t$ where $t=0$ or 1 , where $r \not \equiv-1(\bmod l)$. Then the block component $S_{\mathcal{B}}$ is Morita equivalent to the algebra $\mathcal{D}_{p+1}$.

Proof. The submodule structure of almost all Young modules can be obtained as in the proof of the previous proposition. The structures coincide with those in Erd2, 3.6] except for the one labelled $(p l-l, l)$ has three Specht quotients, namely $(p l, 0)$ and $(p l-1,1)$ and $(p l-l, l)$. By the same argument as in [Erd2] we see that it has the same structure as the module denoted by $E_{3}$ in [Erd2, (3.6)] and the results follows.

Proposition (C). Let $p=2$ and let $S=S_{q}(2, r)$ and $S_{\mathcal{B}}$ be the component corresponding to the principal block.

(a) If $r=2 l+t$ where $0 \leq t<l-1$, then $S_{\mathcal{B}}$ is Morita equivalent to $\mathcal{D}_{3}$.

(b) If $r=2 l+l-1$, then $S_{q}(2, r)$ has a block which is Morita equivalent to $\mathcal{D}_{3}$.

(c) If $r=3 l+t$ where $t=0,1$, then $S_{\mathcal{B}}$ is Morita equivalent to the algebra $\mathcal{S}$ (see §2.7).

Proof. (a) This is a special case of Proposition 3.3(B).

(b) Take the block component which corresponds to core $(l-2,1)$. This is Morita equivalent to the principal block component of $S_{q}(2, r-2)$ as in $\S 3.2$.

(c) We consider the case when $r=3 l$; the other is similar. form

From 3.1 we get that the decomposition matrix for the principal block is of the

$$
\left(\begin{array}{c}
{[\Delta(3 l): L(\mu)]} \\
{[\Delta(3 l-1,1): L(\mu)]} \\
{[\Delta(2 l, l): L(\mu)]} \\
{[\Delta(2 l-1, l+1): L(\mu)]}
\end{array}\right)=\left(\begin{array}{llll}
1 & 1 & 0 & 1 \\
0 & 1 & 1 & 1 \\
0 & 0 & 1 & 1 \\
0 & 0 & 0 & 1
\end{array}\right)
$$

The tilting modules $T(3 l)$ and $T(3 l-1,1)$ are both projective and injective and are isomorphic to to $P(2 l-1, l+1)$ and $P(2 l, l)$ respectively (see [CE 6.4]). The results follows by Proposition 2.7 .

Proposition (D). Let $p=3$ and let $S_{\mathcal{B}}$ be the component of $S:=S_{q}(2, r)$ corresponding to the principal block.

(a) If $r=3 l+t$ where $0 \leq t<l-1$, then $S_{\mathcal{B}}$ is Morita equivalent to $\mathcal{D}_{4}$.

(b) If $r=3 l+l-1$ and $l \neq 2$, then $S$ has a block which is Morita equivalent to $\mathcal{D}_{4}$.

(c) If $r=4 l$, or if $r=4 l+1$ and $l>2$, then $S_{\mathcal{B}}$ is wild. 
Proof. (a) The case $t=0,1$ was done in Proposition 3.3(B), the other $t$ are similar. For (b), take the block with core $(l-2,1)$; see $\S 3.2$.

(c) First we consider the case when $r=4 l$. The decomposition matrix for the principal block component is given by

$$
\left(\begin{array}{c}
{[\Delta(4 l): L(\mu)]} \\
{[\Delta(4 l-1,1): L(\mu)]} \\
{[\Delta(3 l, l): L(\mu)]} \\
{[\Delta(3 l-1, l+1): L(\mu)]} \\
{[\Delta(2 l, 2 l): L(\mu)]}
\end{array}\right)=\left(\begin{array}{lllll}
1 & 1 & 0 & 1 & 1 \\
0 & 1 & 1 & 1 & 0 \\
0 & 0 & 1 & 1 & 0 \\
0 & 0 & 0 & 1 & 1 \\
0 & 0 & 0 & 0 & 1
\end{array}\right)
$$

In fact, from [Th, 3.4, 3.9], $\Delta(4 l-1,1)$ is uniserial with composition factors $L(4 l-1,1), L(3 l-1, l+1), L(3 l, l)$, and $\Delta(4 l)$ has a simple socle isomorphic to $L(3 l-1, l+1)$ and (of course) a simple top, isomorphic to $L(4)$, and

$$
\operatorname{rad} \Delta(4 l) / \operatorname{soc} \Delta(4 l)
$$

is the direct sum of $L(2 l, 2 l)$ and $L(4 l-1,1)$. This shows that the quiver has a "circuit" of length four and an extra edge. Therefore, the algebra is wild by Proposition 2.3(B).

The case $r=4 l+1$ but $l \neq 2$ uses the same argument; the decomposition matrix where the columns and rows are indexed by the partitions $(4 l+1),(4 l-1,2),(3 l+$ $1, l),(3 l-2, l+2)$ and $(2 l+1,2 l)$ have the same entries as in the $r=4 l$ case.

3.4. Proof of Theorem 1.3(A). We can now present a complete proof of Theorem 1.3(A). Recall that we assume $q \neq 1$. Also, if $q$ is a primitive $l$ th root of unity and the characteristic of the field is $p>0$, then $l$ and $p$ are coprime.

We will show that the algebras listed in Theorem 1.3(A) are semi-simple, and to complete the proof we will show that the algebras listed in Theorem $1.4(\mathrm{~A})$ are not semi-simple.

(3.4.1) [1.3(i) and 1.3(ii)]. If $q$ is not a root of unity, or $q$ is an lth root of unity and $r<l$, then $S_{q}(n, r)$ is semi-simple:

The Hecke algebra is semisimple when $q$ is not a root of unity or when $q$ is a primitive $l$ th root of unity and $r<l$ by a result of Dipper and James [DJ2]. Therefore, $S_{q}(n, r)$ is semisimple because the endomorphism algebra of a completely reducible module is semisimple.

From now we assume that $q$ is a primitive $l$ th root of 1 .

(3.4.2) [1.3(iii) and 1.4(d)]. Let $l=2$ and $r=2 w+1$. If $p=0$, then $S_{q}(2, r)$ is semi-simple. Moreover, if $p \geq 3$, then $S_{q}(2, r)$ is semisimple if and only if $w<p$.

Let $p=0$, the first part of the statement holds by Proposition 3.3(A)(a) and the results in $\S 3.2$. Let $p \geq 3$. Then $S_{q}(2, r)$ has two block components, by $\S 3.1$. We know that the prinicpal block component is Morita equivalent to the classical Schur algebra $S(2, w)$ and this is semisimple if and only if $w<p$ (see [DN]). So for $w \geq p$ the algebra $S_{q}(2, r)$ is not semisimple. Now let $w<p$; the other block component of $S_{q}(2, r)$ is Morita equivalent to the principal block component of $S_{q}(2, r-2)$, hence Morita equivalent to $S(2, w-1)$, and we get that for $w<p$ the algebra $S_{q}(2, r)$ is semisimple.

Now assume $r \geq l$.

(3.4.3) [1.4(b) and most of $1.4(\mathrm{a})]$. If $l \geq 3$ and $n \geq 2$, then $S_{q}(n, r)$ is not semi-simple for $r \geq l$.

Consider $S_{q}(2, l)$ and $S_{q}(2, l+1)$ for $l \geq 3$. These algebras are not semisimple by Proposition 3.3(A)(b). Therefore, $S_{q}(2, r)$ is not semisimple for $r \geq l$ by Proposition 
2.4(B), and $S_{q}(n, r)$ is not semisimple for $l \geq 3, n \geq 2$, and $r \geq l$ by Proposition $2.4(\mathrm{~A})$.

(3.4.4) [1.4(a)]. If $l=2$ and $n \geq 3$, then $S_{q}(n, r)$ is not semisimple for $r \geq l$.

By Proposition 3.3(A)(b) we know that $S_{q}(2,2)$ and $S_{q}(2,4)$ are not semisimple, hence $S_{q}(3,2)$ and $S_{q}(3,4)$ are not semisimple. Moreover, $S_{q}(3,3)$ is not semisimple because $\Delta(3)$ is not simple (but indecomposable). Hence, $S_{q}(n, r)$ is not semisimple for $n \geq 3$ and $r \geq l$ by Propositions 2.4(A) and 2.4(B).

(3.4.5) $[1.4(\mathrm{c})]$. If $l=2$, then $S_{q}(2, r)$ is not semisimple for $r=2 w \geq 2$ where $w \in \mathbb{N}$.

Consider $S_{q}(2,2)$ for $l=2$. This algebra is not semisimple by Proposition 3.3(A)(b). Therefore, $S_{q}(2, r)$ is not semisimple for $r=2 w \geq 2$ where $w \in \mathbb{N}$ by Proposition 2.4(B).

\section{Representation type FOR $S_{q}(n, r), n \geq 3$}

4.1. Let $l \geq 3$ and consider the principal block component of the algebra $S_{q}(3,2 l)$. We first claim that some of the decomposition numbers for Weyl modules are given by the following matrix:

$$
\left(\begin{array}{c}
{[\Delta(2 l): L(\mu)]} \\
{[\Delta(2 l-1,1): L(\mu)]} \\
{[\Delta(l, l): L(\mu)]} \\
{\left[\Delta\left(2 l-2,1^{2}\right): L(\mu)\right]} \\
{[\Delta(l, l-1,1): L(\mu)]} \\
{\left[\Delta\left((l-1)^{2}, 2\right): L(\mu)\right]}
\end{array}\right)=\left(\begin{array}{cccccc}
1 & 1 & 0(1) & 0 & 0 & 1 \\
0 & 1 & * & * & * & * \\
0 & 0 & 1 & * & * & * \\
0 & 0 & 0 & 1 & 1 & 0 \\
0 & 0 & 0 & 0 & 1 & 1 \\
0 & 0 & 0 & 0 & 0 & 1
\end{array}\right)
$$

If $p \neq 2$, then the Weyl module $\Delta(2 l)$ has length three and has the stated composition factors. Moreover, this module is uniserial with second socle $L(2 l-1,1)$ for $p \neq 2$. For $p=2$ there is an extra composition factor $L(l, l)$, and it occurs in the second radical layer. In both cases, $\Delta(2 l)$ contains a uniserial submodule of length two with top $L(2 l-1,1)$ and socle $L\left((l-1)^{2}, 2\right)$. The last three rows follow easily by consideration of dimensions, and by using Steinberg's tensor product theorem.

Lemma. Let $l \geq 3$. Then the prinicpal block component of $S_{q}(3,2 l)$ is wild.

Proof. To see this we investigate the quiver. Consider the Schur algebra $S_{q}(3, l)$ over the same field. The principal block component has the Weyl module $\Delta(l-1,1)$, of length two, with socle $L\left(l-2,1^{2}\right)$. By tensoring with $L(1)^{F}$ we get an indecomposable module of length two with socle $L\left(2 l-2,1^{2}\right)$ and head $L(2 l-1,1)$. Using this and the information from the last three rows of the decomposition matrix together with the above-mentioned submodule of $\Delta(2 l)$ we see that the quiver contains a circuit with four vertices. Since the quiver is connected with 6 vertices, it contains this as a proper subquiver and hence the algebra is wild by Proposition 2.3(B).

Proposition (E). If $l \geq 4$, then $S_{q}(3, r)$ has wild representation type for $r=2 l$, $2 l+1$ and $2 l+2$.

Proof. We have seen that the block component of $S_{q}(3,2 l)$ containing $L\left(2 l-2,1^{2}\right)$ has wild representation type. Consider the blocks of $S_{q}(3,2 l+1)\left(\right.$ resp. $S_{q}(3,2 l+2)$ ) which contain the simple modules $L(2 l-2,2,1)$ (resp. $L\left(2 l-2,2^{2}\right)$ ). Set $\lambda=$ $\left(2 l-2,1^{2}\right), \mu_{1}=(2 l-2,2,1)$ and $\mu_{2}=\left(2 l-2,2^{2}\right)$. The weights $\lambda, \mu_{1}$ and $\mu_{2}$ belong to the same facet for $l \geq 4$ and $\lambda-\mu_{j} \in P(D)$ for $j=1,2$. Consequently, 
by Proposition $2.4(\mathrm{C})$, it follows that $S_{q}(3,2 l+1)$ and $S_{q}(3,2 l+2)$ have wild representation type.

4.2. We now provide a list of algebras for $n=3,4,5$ along with their representation type. This is verified on a case-by-case basis in Sections 5 and 6 .

(4.2.1) $S_{q}(3,7), p \neq 2, l=3$ (tame, $\left.\S 5.1\right)$,

(4.2.2) $S_{q}(3,8), p \neq 2, l=3$ (tame, §5.1),

(4.2.3) $S_{q}(3,10), p \geq 0, l=3$, (wild, §5.2),

(4.2.4) $S_{q}(3,11), p \geq 0, l=3$, (wild, §5.2),

(4.2.5) $S_{q}(3,7), p=2, l=3$ (wild, $\left.§ 5.3\right)$,

(4.2.6) $S_{q}(3,8), p=2, l=3$ (wild, §5.3),

(4.2.7) $S_{q}(3,4), p \geq 0, l=2$, (tame, $\left.\S 5.4\right)$,

(4.2.8) $S_{q}(3,5), p \geq 0, l=2$, (tame, 55.5$)$,

(4.2.9) $S_{q}(3,6), p \geq 0, l=2$, (wild, §5.6),

(4.2.10) $S_{q}(3,7), p \geq 0, l=2$, (wild, §5.7),

(4.2.11) $S_{q}(3,8), p \geq 0, l=2$, (wild, $\left.§ 5.8\right)$,

(4.2.12) $S_{q}(4,7), p \neq 2, l=3$, (wild, $\S 6.1$ ),

(4.2.13) $S_{q}(4,8), p \neq 2, l=3$, (wild, $\left.\S 6.2\right)$,

(4.2.14) $S_{q}(4,4), p \geq 0, l=2$, (wild, $\left.\S 6.3\right)$,

(4.2.15) $S_{q}(4,5), p \geq 0, l=2$, (tame, $\left.\S 6.4\right)$,

(4.2.16) $S_{q}(5,5), p \geq 0, l=2$, (wild, $\left.\S 6.5\right)$.

4.3. Proof of Theorem 1.3(B). Assume now that $S_{q}(n . r)$ is not semisimple. That is, $q$ is a primitive $l$ th root of unity and $r \geq l$. Moreover, if $n=2$ and $l=2$, then either $r$ is even, or $p \geq 3$ and $r \geq 2 p+1$. We will show that the algebras listed in Theorem 1.3(B) have finite type, and that the algebras listed in Theorem $1.4(\mathrm{~B})$ are of infinite type. Note that in the following the semisimple algebras are excluded.

(4.3.1) $[1.3(\mathrm{i})]$. If $r<2 l$, then $S_{q}(n, r)$ has finite representation type.

If $r<2 l$, then all blocks of $\mathcal{H}_{q}(r)$ are Brauer tree algebras of finite type, or are semisimple $([\mathrm{U}]$, see $[\mathrm{EN}])$. The proof of [Erd2, (4.1)] works here as well and we deduce that $S_{q}(n, r)$ has finite type.

(4.3.2) [1.3(ii) and part of $1.3(\mathrm{iv})]$. If $p \neq 0$, then $S_{q}(2, r)$ has finite representation type for $r<l p$.

Let $l \geq 2$. As explained in $\S 3.2$, every block component of $S_{q}(2, r)$ is Morita equivalent to a principal block component of some $S_{q}(2, d)$ for some $d \leq r$ [Don]. Let $d=w l+t \leq r$ where $0 \leq w$ and $0 \leq t \leq l-1$, so that $w<p$. By Proposition $3.3(\mathrm{~A})$ we have that the principal block component of $S_{q}(2, d)$ is semi-simple or of finite type. It follows that $S_{q}(2, r)$ has finite type for $r<l p$.

(4.3.3) $[1.3(\mathrm{iii})]$. If $p=0$, then $S_{q}(2, r)$ has finite representation type (if it is not semi-simple).

This follows from Proposition 3.3(A) and the results in $\S 3.2$.

(4.3.4) [rest of $1.3(\mathrm{iv})$ and part of $1.4(\mathrm{c})]$. If $p \neq 0$ and $l=2$, then $S_{q}(2, r)$ has infinite representation type for $r \geq 2 p^{2}+1$ and has finite representation type for $r=2 w+1$ where $p \leq w<p^{2}$.

Let $r=2 w+1$ and $p \leq w$. For $l=2$, we have from $\S 3.2$ that the principal block component of $S_{q}(2, r)$ is Morita equivalent to the classical Schur algebra $S(2, w)$, over characteristic $p$. The other block component is Morita equivalent to the principal block component of $S_{q}(2, r-2)$. Therefore, by Erd2 $S_{q}(2,2 w+1)$ 
has finite representation type if $w \in\left\{1,2, \ldots, p^{2}-1\right\}$, and infinite representation type if $w \geq p^{2}$. Now apply Proposition 2.3(B).

(4.3.5) [1.4(a)]. If $n \geq 3$ and $r \geq 2 l$, then $S_{q}(n, r)$ has infinite representation type.

By Proposition 2.4(A) and Proposition 2.4(B) it suffices to prove this for $n=3$ and $r=2 l, 2 l+1$ and $r=2 l+2$. This holds by Proposition 4.1 for $l \geq 4$, and by Lemma 4.1, (4.2.1),(4.2.2), (4.2.5), and (4.2.6) for $l=3$, and by (4.2.7), (4.2.8) and (4.2.9) when $l=2$.

(4.3.6) $[1.4(\mathrm{~b})]$. If $l \geq 3$ and $p \neq 0$, then $S_{q}(2, r)$ has infinite representation type for $r \geq l p$.

Let $p \geq 2$. By Proposition 3.3(B) the algebra $S_{q}(2, l p)$ has infinite type, for $l \geq 2$. If $l \geq 3$, then also $S_{q}(2, l p+1)$ has infinite type, by Proposition 2.4(C) and hence for $l \geq 3$ and any $r \geq l p$ the algebra $S_{q}(2, r)$ has infinite type.

(4.3.7) [rest of 1.4(c)]. If $p \geq 3, l=2$ and $r$ is even, then $S_{q}(2, r)$ has infinite representation type for $r \geq 2 p$.

The algebra $S_{q}(2,2 p)$ has infinite representation type by Proposition 3.3(B); now apply Proposition 2.3(B).

4.4. Proof of Theorem 1.3(C). We assume that $S_{q}(n, r)$ is of infinite type, so that one of the conditions in Theorem 1.4(B) holds. We show that the algebras listed in Theorem 1.3(C) are tame, and moreover, that the algebras in Theorem $1.4(\mathrm{C})$ are wild.

(4.4.1) [1.3(i-iii)]. $S_{q}(n, r)$ has tame representation type for $n=3, l=3, p \neq 2$ and $r=7,8 ; n=3, l=2, p \geq 0$ and $r=4,5 ; n=4, l=2, p \geq 0$ and $r=5$.

The tameness for $S_{q}(n, r)$ for $n=3, l=3, p \neq 2$ and $r=7,8$ holds by (4.2.1) and (4.2.2); for $n=3, l=2, p \geq 0$ and $r=4,5$ by (4.2.7) and (4.2.8); for $n=4$, $l=2, p \geq 0$ and $r=5$ by $(4.2 .15)$.

(4.4.2) [1.3(iv)]. $S_{q}(2, r)$ has tame representation type for $l \geq 3, p=2,3$ and $p l \leq r<(p+1) l$.

We can apply Proposition 3.3(C) and Proposition 3.3(D), together with the results from $\S 3.2$. The algebras involved are Morita equivalent to either $\mathcal{D}_{3}$ or $\mathcal{D}_{4}$ which are tame by [DEMN, $\S 5.1, \S 5.5]$.

(4.4.3) [1.3(v) and part of 1.4(e)]. If $p=3, l=2$ (so that $r \geq 6$ ), then $S_{q}(2, r)$ is tame for $r \in\{6,19,21,23\}$ and wild for $r$ even and $r \geq 8$ and for $r$ odd and $r \geq 25$.

If $r$ is even, then $S_{q}(2, r)$ is tame for $r=6$, by Proposition 3.3(D) and it is wild for $r=8$ and hence wild for all even $r \geq 8$. Now let $r=2 w+1$; by $\S 3.2$, the principal block component of $S_{q}(2,2 w+1)$ is Morita equivalent to the classical Schur algebra $S(2, w)$ and the other block component is Morita equivalent to the classical Schur algebra $S(2, w-1)$. By [DEMN], over characteristic 3 the classical Schur algebra $S(2, m)$ is tame if and only if $m=9,10,11$, it is wild for $m \geq 12$ and of finite type for $m \leq 8$.

To complete the proof we must show that the remaining algebras are wild. Assume now that $2 l \leq r$.

(4.4.4) [1.4(a)]. Assume $n \geq 3$ and $l \geq 4$, then $S_{q}(n, r)$ is wild.

By Proposition 2.3(B) and Proposition 2.3(A) it suffices to show this for $n=3$ and $r=2 l, 2 l+1,2 l+2$. This was verified in Proposition 4.1.

(4.4.5) [1.4(b)]. Assume that $n \geq 3$ and $l=3$. Then $S_{q}(n, r)$ is wild unless $p \neq 2$ and $r=7,8$. 
Consider first the case $p \neq 2$. If $r=6$ and $n=3$, then by Lemma 4.1 the algebra is wild, and, then also for $r=3 k \geq 6$. If $r=10,11$ and $n=3$, then by (4.2.3) and (4.2.4) the algebra is wild. Combining these it follows, using Propositions 2.4(A) and 2.4(B), that for all $n \geq 3$ and $r \geq 9$, the algebra $S_{q}(n, r)$ is wild. If $n=4$ and $r=7,8$, then by (4.2.12) and (4.2.13) the algebra is wild. Together with the previous result we deduce that $S_{q}(n, r)$ is wild for $n \geq 4$ and $r \geq 6$.

Now assume $p=2$. Then $S_{q}(3, r)$ is wild for $r=6,7,8$ by Lemma 4.1 , (4.2.5) and (4.2.6). Using Propositions 2.3(A) and 2.3(B) it follows that $S_{q}(n, r)$ is wild for $n \geq 3$ and $r \geq 6$ when $p=2$.

(4.4.6) $[1.4(\mathrm{c})]$. Let $n \geq 3$ and $l=2$ (so that $r \geq 4$ ). Then $S_{q}(n, r)$ is wild unless $n=3$ and $r=4$, or $3 \leq n \leq 4$ and $r=5$.

If $n=4$ and $r=4$, then the algebra is wild by (4.2.14), and if $n=5, r=5$ it is wild by (4.2.16). Now suppose $n=3$ and $r=6,7,8$, then the algebra is wild by (4.2.9), (4.2.10), and (4.2.11). These imply 1.4(c), using Propositions 2.3(A) and $2.3(\mathrm{~B})$.

(4.4.7) $[1.4(\mathrm{~d})]$. Let $n=2$ and $l \geq 3$ (so that $r \geq p l, p \neq 0$ ). Then $S_{q}(2, r)$ is wild unless $p \leq 3$ and $p l \leq r<(p+1) l$.

If $p \geq 5$, then apply Proposition 3.3(B) which shows that $S_{q}(2, r)$ is wild for $r \geq l p$. For $p=2$ and $r=3 l, 3 l+1$ use Proposition 3.3(C) and Proposition 2.7, so $S_{q}(2, r)$ is wild for $r \geq 3 l$. Suppose $p=3$, then use Proposition 3.3(D) and it follows that $S_{q}(2, r)$ is wild for $r \geq 4 l$.

(4.4.8) [rest of 1.4(e)]. Let $l=2$ and $n=2$, and $p \neq 0$ and assume that either $r$ is even $\geq 2 p$, or $r$ is odd $\geq 2 p^{2}+1$. Then $S_{q}(2, r)$ is wild unless $p=3$ and $r \in\{6,19,21,23\}$.

By Proposition 3.3(B) the algebra is wild for $p \geq 5$ and $r=4,5$, As explained in (4.4.3) it is wild for $p=3, r$ even $\geq 8$ and for $r$ odd, $\geq 25$.

\section{Representation type For some $S_{q}(3, r)$}

5.1. In the next three sections we will deal with algebras $S_{q}(3, r)$ where $l=3$. First we consider the case when the characteristic of the field is not two. We recall the definition of the algebras $\mathcal{R}_{4}$ and $\mathcal{H}_{4}$. The algebra $\mathcal{R}_{4}$ is given by the quiver in Figure 4 with relations

$$
\alpha_{1} \beta_{1}=0, \quad \alpha_{1} \alpha_{2}=0=\beta_{2} \beta_{1}, \quad \beta_{1} \alpha_{1}=\alpha_{2} \beta_{2}, \quad \beta_{2} \alpha_{2}=\alpha_{3} \beta_{3} .
$$

The algebra $\mathcal{H}_{4}$ has quiver in Figure 5 and is defined by the following relations:

$$
\begin{aligned}
& \alpha \delta=0=\gamma \beta, \quad \alpha \beta=0=\alpha \rho \\
& \zeta \beta=0=\zeta \rho, \quad \delta \gamma=\beta \alpha+\rho \zeta
\end{aligned}
$$

Proposition. Let $p \neq 2$ and $l=3$. The basic algebra of the principal block of $S_{q}(3, r)$ is Morita equivalent to $\mathcal{R}_{4}$ [Erd2, 3.11] if $r=7$ and $\mathcal{H}_{4}$ [Erd2, 3.13] if

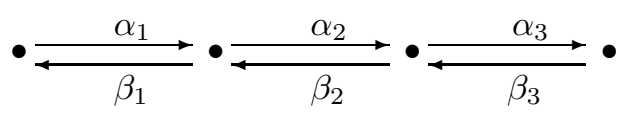

Figure 4. 


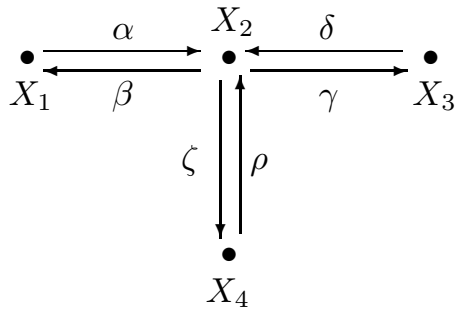

Figure 5 .

$r=8$. Furthermore, under these conditions the algebras $S_{q}(3,7)$ and $S_{q}(3,8)$ are tame.

Proof. First let $r=7$ and consider the principal block component. The aim is to show that the hypotheses for [Erd2, 3.12] are satisfied for the Young modules. To get the filtration multiplicities for the Young modules by reciprocity (see $\S 2.6$ ), we start by finding the decomposition matrix for the Weyl modules. We claim that it is of the form

$$
\left(\begin{array}{c}
{[\Delta(7): L(\mu)]} \\
{[\Delta(5,2): L(\mu)]} \\
{[\Delta(4,3): L(\mu)]} \\
{[\Delta(4,2,1): L(\mu)]}
\end{array}\right)=\left(\begin{array}{llll}
1 & 1 & 0 & 0 \\
0 & 1 & 1 & 1 \\
0 & 0 & 1 & 1 \\
0 & 0 & 0 & 1
\end{array}\right) .
$$

The first row can be obtained from [Th]. By row or column removal one gets the third row and also $[\Delta(5,2): L(4,3)]=1$. Then there is only one missing entry which follows by consideration of dimensions.

Next we will show that the decomposition matrix for 3 -part partitions of $\mathcal{H}_{q}(7)$ when $l=3$ (and $p \neq 2$ ) is given as follows:

$$
\left(\begin{array}{c}
{\left[S^{(7)}: D^{\mu}\right]} \\
{\left[S^{(5,2)}: D^{\mu}\right]} \\
{\left[S^{(4,3)}: D^{\mu}\right]} \\
{\left[S^{(4,2,1)}: D^{\mu}\right]}
\end{array}\right)=\left(\begin{array}{cccc}
1 & 0 & 0 & 0 \\
1 & 1 & 0 & 0 \\
0 & 1 & 1 & 0 \\
1 & 1 & 1 & 1
\end{array}\right) .
$$

First we know that $Y^{(5,2)}$ has Specht quotients $S^{(5,2)}$ and $S^{(7)}$ which gives the second row. Next we have $\left[S^{(4,3)}: D^{(5,2)}\right]=1$ by column removal and the decomposition matrix for $\mathcal{H}_{q}(3)$. We see that $D^{(4,3)}$ is 1 -dimensional and that by dimensions, the first entry of the row of $\left[S^{(4,3)}: D^{\mu}\right]$ must be 0 .

By row removal we get $\left[S^{(4,2,1)}: D^{(4,3)}\right]=1$. Moreover, $Y^{(4,2,1)}$ is projective and must be $P\left(D^{(5,2)}\right)$ and hence by reciprocity $\left[S^{(4,2,1)}: D^{(5,2)}\right]=1$. The Specht module $S^{\left(4,1^{3}\right)}$ is irreducible (see [Jam, 6.22] ), and is isomorphic to $D^{\alpha}$ for some $\alpha \triangleright\left(4,1^{3}\right)$. Moreover, this module has dimension 20 and the only possibility is $\alpha=(4,2,1)$. This gives also the last entry, that is $\left[S^{(4,2,1)}: D^{(7)}\right]$, by dimensions.

By reciprocity we see that $Y^{(4,3)}$ has Specht quotients $S^{(5,2)}$ and $S^{(4,3)}$ and since it is self-dual, it must have a simple top $D^{(5,2)}$. Hence $Y^{(4,3)}$ is a quotient of $P(5,2)$ and the kernel is necessarily equal to $S^{(4,2,1)}$ (one inclusion since $D^{(4,2,1)}$ does not occur and equality by length). This gives the second radical layer of $P(5,2)$, which is $k \oplus D^{(4,3)}$. It follows that $S^{(4,2,1)}$ has "diamond shape" with socle $D^{(5,2)}$. The dimensions of $\operatorname{Hom}_{\mathcal{H}_{q}(7)}\left(Y^{\lambda}, Y^{\mu}\right)$ are characteristic-independent (see [DJ1]), and are seen by considering the Specht-quotients. We get the remaining hypothesis for Erd2 3.12] and deduce that the block component of $S_{q}(3,7)$ is Morita equivalent 
to $\mathcal{R}_{4}$, as stated. There are two more blocks which are both of finite type (which is easy to see). Hence, $S_{q}(3,7)$ is tame.

Now consider the algebra $S_{q}(3,8)$ for $l=3$ where $p \neq 2$. The aim is to verify the hypotheses of [Erd2, 3.14] for the principal block component. The decomposition matrix for the Weyl modules is

$$
\left(\begin{array}{c}
{[\Delta(8): L(\mu)]} \\
{[\Delta(5,3): L(\mu)]} \\
{[\Delta(5,2,1): L(\mu)]} \\
{[\Delta(4,3,1): L(\mu)]}
\end{array}\right)=\left(\begin{array}{llll}
1 & 0 & 1 & 0 \\
0 & 1 & 1 & 1 \\
0 & 0 & 1 & 1 \\
0 & 0 & 0 & 1
\end{array}\right)
$$

The verification is similar to the $S_{q}(3,7)$ case. It follows that the Young modules corresponding to the partitions $(8)$ and $(5,3)$ are simple and hence so are the corresponding Specht modules. The Young module $Y^{(5,2,1)}$ has a "cross shape" (cf. Erd2 3.14]). This gives the decomposition numbers for the Specht module $S^{(5,2,1)}$. Moreover, $Y^{(4,3,1)}=P\left(D^{(5,3)}\right)$. The decomposition matrix for 3-part partitions of the Hecke algebra is therefore given by

$$
\left(\begin{array}{c}
{\left[S^{(8)}: D^{\mu}\right]} \\
{\left[S^{(5,3)}: D^{\mu}\right]} \\
{\left[S^{(5,2,1)}: D^{\mu}\right]} \\
{\left[S^{(4,3,1)}: D^{\mu}\right]}
\end{array}\right)=\left(\begin{array}{cccc}
1 & 0 & 0 & 0 \\
0 & 1 & 0 & 0 \\
1 & 1 & 1 & 0 \\
* & 1 & 1 & 1
\end{array}\right)
$$

Note that the multiplicity of $D^{(5,2,1)}$ in $S^{(4,3,1)}$ is seen by column removal. The Specht module $S^{(4,3,1)} \subset P\left(D^{(5,3)}\right)$ must be contained in the square of the radical. Then it follows that the remaining decomposition number is zero and that the submodule structure of $P\left(D^{(5,3)}\right)$ is as needed for [Erd2, 3.14].

Therefore, one can conclude that the principal block of $S_{q}(3,8)$ is Morita equivalent to the algebra $\mathcal{H}_{4}$ as in Erd2]. This has tame representation type by [DEMN] 5.7]. For the representation type of the entire algebra one must also consider the other blocks. The block containing $L\left(6,1^{2}\right)$ is Morita equivalent to $\mathcal{A}_{2}$. This leaves us with the block containing $L(7,1)$. By the same arguments as for $S_{q}(3,7)$ (in the first part of the proof) one shows that this block component is Morita equivalent to the algebra $\mathcal{R}_{4}$, and it follows that $S_{q}(3,8)$ is tame for $l=3$ and $p \neq 2$.

5.2. We will now consider the algebras $S_{q}(3,10)$ and $S_{q}(3,11)$ for $l=3$. The following two results show that these algebras of wild representation type areindependent of the characteristic of the field.

Proposition (A). The algebra $S_{q}(3,10)$ is wild when $l=3$.

Proof. If $p=2$ and $l=3$, then by Proposition 3.3(C), $S_{q}(2,10)$ is wild, hence $S_{q}(3,10)$ is wild by Proposition 2.4(A). So assume that $p \neq 2$. The decomposition matrix for the Weyl modules in the principal block component is given as follows:

$$
\left(\begin{array}{c}
{[\Delta(10): L(\mu)]} \\
{[\Delta(8,2): L(\mu)]} \\
{[\Delta(7,3): L(\mu)]} \\
{[\Delta(7,2,1): L(\mu)]} \\
{[\Delta(5,5): L(\mu)]} \\
{\left[\Delta\left(4,3^{2}\right): L(\mu)\right]}
\end{array}\right)=\left(\begin{array}{llllll}
1 & 1 & 0 & 0 & 0 & 0 \\
0 & 1 & 1 & 1 & 0 & 0 \\
0 & 0 & 1 & 1 & 1 & 1 \\
0 & 0 & 0 & 1 & 0 & 1 \\
0 & 0 & 0 & 0 & 1 & 1 \\
0 & 0 & 0 & 0 & 0 & 1
\end{array}\right)
$$

The tilting modules $T(5,5)$ and $T(7,2,1)$ are both uniserial of length three, with top and socle $L(4,3,3)$ and the middle is $L(5,5)$ or $L(7,2,1)$ respectively. So they 
are both quotients of $P(4,3,3)$. We deduce that there is an exact sequence

$$
0 \rightarrow W=\Delta(7,3) \rightarrow P\left(4,3^{2}\right) \rightarrow X \rightarrow 0
$$

and an exact sequence

$$
0 \rightarrow \Delta(5,5) \oplus \Delta(7,2,1) \rightarrow X \rightarrow \Delta(4,3,3) \rightarrow 0
$$

Let $U$ be the quotient of $P\left(4,3^{2}\right)$ by $\operatorname{rad}(W)$. We claim that the socle of $U$ is $L(7,3) \oplus L\left(4,3^{2}\right) \oplus L\left(4,3^{2}\right)$. This is clear from the above should it be the case that

$$
\operatorname{Ext}_{S_{q}(3,10)}^{1}\left(L(7,3), L\left(4,3^{2}\right)\right)=0 \text {. }
$$

Otherwise, $L(7,3)$ must occur in the top of the radical of $P\left(4,3^{2}\right)$ and then it also lies in the socle of $U$. This is enough to see that the algebra is wild. Namely, let $e=e_{\left(4,3^{2}\right)}+e_{(7,3)}$ where the $e_{\lambda}$ are orthogonal primitive, with $e_{\lambda} S_{q}(3,10)=P(\lambda)$, and apply Proposition 2.3(A).

Proposition (B). The algebra $S_{q}(3,11)$ is wild when $l=3$.

Proof. If $p=2$, then $S_{q}(2,11)$ is wild, so $S_{q}(3,11)$ is wild. Let $p \neq 2$. The decomposition matrix for the principal block component is

$$
\left(\begin{array}{c}
{[\Delta(11): L(\mu)]} \\
{[\Delta(8,3): L(\mu)]} \\
{[\Delta(8,2,1): L(\mu)]} \\
{[\Delta(7,3,1): L(\mu)]} \\
{\left[\Delta\left(5^{2}, 1\right): L(\mu)\right]} \\
{\left[\Delta\left(5,3^{2}\right): L(\mu)\right]}
\end{array}\right)=\left(\begin{array}{llllll}
1 & 0 & 1 & 0 & 0 & 0 \\
0 & 1 & 1 & * & * & * \\
0 & 0 & 1 & 1 & 0 & 0 \\
0 & 0 & 0 & 1 & 1 & 1 \\
0 & 0 & 0 & 0 & 1 & 1 \\
0 & 0 & 0 & 0 & 0 & 1
\end{array}\right)
$$

The lower part of the matrix is obtained from the decomposition matrix of $S_{q}(3,8)$ over the same field by tensoring with the determinant.

The block of $S_{q}(3,8)$ containing $L(7,1)$ is known, it is Morita equivalent to the algebra $\mathcal{R}_{4}$. This together with the structure of $\Delta(11)$ shows that the quiver contains a subquiver

$$
L(11) \longleftrightarrow L(8,2,1) \longleftrightarrow L(7,3,1) \longleftrightarrow L\left(5^{2}, 1\right) \longleftrightarrow L\left(5,3^{2}\right)
$$

In fact, this is the full subquiver with these vertices. Assume (for a contradiction) that the algebra is not wild; then the vertex $L(8,3)$ cannot be joined to too many other simples by $2.3(\mathrm{~B})$. Since there exists a module with simple top and $\Delta$ quotients $\Delta(8,2,1), \Delta(8,3)$ (the quotient $P(8,2,1) / \Delta(11))$, it follows that $L(8,3)$ must be joined to $L(8,2,1)$ or $L(7,3,1)$ in the quiver; so that by $2.3(\mathrm{~B})$ it is joined to precisely one of these and not to any other vertex of the quiver. Therefore, this gives the quiver completely.

Suppose the first possibility holds, then by considering dimensions and using the fact that $\Delta(8,3)$ has a simple top, it follows that $\Delta(8,3)$ can only be uniserial, and its composition factors must be

$$
L(8,3), L(8,2,1), L(7,3,1), L\left(5^{2}, 1\right) .
$$

This implies that $P(8,2,1)$ has a uniserial quotient of length three with composition factors $L(8,2,1), L(7,3,1), L\left(5^{2}, 1\right)$. All the weights $\mu$ occurring are $\leq(8,2,1)$, so this must be a quotient of the Weyl module $\Delta(8,2,1)$, a contradiction. 


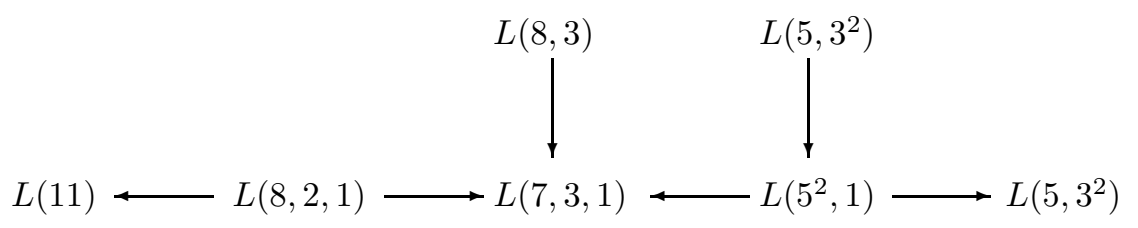

FiguRE 6.

Now consider the second possibility. Recall from the structure of $\mathcal{R}_{4}$ that $P\left(5,3^{2}\right)$ has a quotient with radical layering

$$
\begin{gathered}
L\left(5,3^{2}\right), \\
L\left(5^{2}, 1\right), \\
L(7,3,1) \oplus L\left(5,3^{2}\right) .
\end{gathered}
$$

Consider the representations of the wild quiver $\widetilde{\widetilde{\mathcal{D}}_{4}}$ given in Ri1]. There is a functor from the category of the representations of this quiver into the module category of the quiver given in Figure 6, and this can be identified with a subcategory of $S_{q}(3,11)$. The functor preserves indecomposables and reflects isomorphisms, hence $S_{q}(3,11)$ is wild.

5.3. Consider $S_{q}(3,7)$ and $S_{q}(3,8)$ for $l=3$ and $p=2$. We will show that both of these algebras are wild. First, we look at $S_{q}(3,7)$. The Weyl module $\Delta(7)$ is uniserial of length three, socle $L(5,2)$; and the decomposition matrix for the principal block component is given by

$$
\left(\begin{array}{c}
{[\Delta(7): L(\mu)]} \\
{[\Delta(5,2): L(\mu)]} \\
{[\Delta(4,3): L(\mu)]} \\
{[\Delta(4,2,1): L(\mu)]}
\end{array}\right)=\left(\begin{array}{llll}
1 & 1 & 1 & 0 \\
0 & 1 & 1 & 1 \\
0 & 0 & 1 & 1 \\
0 & 0 & 0 & 1
\end{array}\right)
$$

We determine now the decomposition matrix for the principal block of the Hecke algebra. By using similar arguments given for $l=3$ and $p \neq 2$ we see that this is of the form

$$
\left(\begin{array}{c}
{\left[S^{(7)}: D^{\mu}\right]} \\
{\left[S^{(5,2)}: D^{\mu}\right]} \\
{\left[S^{(4,3)}: D^{\mu}\right]} \\
{\left[S^{(4,2,1)}: D^{\mu}\right]}
\end{array}\right)=\left(\begin{array}{llll}
1 & 0 & 0 & 0 \\
1 & 1 & 0 & 0 \\
0 & 1 & 1 & 0 \\
1 & 1 & 1 & 1
\end{array}\right)
$$

We return to the $q$-Schur algebra. The projective $P(4,2,1)$ is a tilting module (isomorphic to $T(5,2)$ ), and hence is also injective, its radical layers are

$$
\begin{gathered}
L(4,2,1), \\
L(4,3), \\
L(5,2) \oplus L(4,2,1), \\
L(4,3), \\
L(4,2,1) .
\end{gathered}
$$

This gives the structure of $\Delta(5,2)$. The tilting module $T(7)$ has quotients $\Delta(7)$, $\Delta(5,2)$ and $\Delta(4,2,1)$. It cannot be $P(4,2,1)$, so it does not have a simple top. 
Hence the top and socle must be a direct sum $L(4,2,1) \oplus L(5,2)$ and we deduce that $T(7)$ is ' $\tilde{D}_{6}^{\prime}$-shaped. The reason why this is important is that it shows that the (unique) non-split extension of $\Delta(5,2)$ and $\Delta(7)$ has a "tree shape" and is isomorphic to $P(5,2)$. The radical layering of $P(5,2)$ is given by

$$
\begin{aligned}
& L(5,2), \\
& L(4,3), \\
& L(7) \oplus L(4,2,1), \\
& L(4,3), \\
& L(5,2) .
\end{aligned}
$$

That is, $L(4,2,1)$ is in the socle and the factor module is uniserial. As for the structure of $P(4,3)$, its radical is a direct sum of $\Delta(7)$ with the second radical of $P(4,2,1)$, and there is an exact sequence

$$
0 \rightarrow \Delta(7) \rightarrow P(4,3) \rightarrow \operatorname{rad}_{S_{q}(3,7)} P(4,2,1) \rightarrow 0 .
$$

The structure of this algebra is similar to that of the algebra in DEMN, 3.6]. It is wild, by a similar argument (which is actually not quite correct in [DEMN, 3.6], the zero relation was ignored but we will prove it here). Consider the quiver given in Figure 7 where the only relation is $\alpha \beta=0$. This is wild, it contracts to $C \widetilde{\widetilde{D}}_{4}$ (see [Ri1 p. 168]).

It is interesting to note that although the decomposition matrices for the 2-part partitions of $\mathcal{H}_{q}(7)$ are the same for $p=2$ and for $p \neq 2$, the Young modules are different. If $p \neq 2$, then $Y^{(4,2)}$ has two Specht quotients but if $p=2$, it has three.

Next consider the algebra $S_{q}(3,8)$ for $l=3$ and $p=2$. Let us focus our attention on the block containing $L(7,1)$. The decomposition matrix for the Weyl modules is

$$
\left(\begin{array}{c}
{[\Delta(7,1): L(\mu)]} \\
{[\Delta(6,2): L(\mu)]} \\
{[\Delta(4,4): L(\mu)]} \\
{\left[\Delta\left(4,2^{2}\right): L(\mu)\right]}
\end{array}\right)=\left(\begin{array}{llll}
1 & 1 & 1 & 0 \\
0 & 1 & 1 & 1 \\
0 & 0 & 1 & 1 \\
0 & 0 & 0 & 1
\end{array}\right) .
$$

We determine as for $S_{q}(3,7)$ (the previous case) the decomposition numbers for the block of the Hecke algebra and use this to determine tilting modules for the $q$-Schur algebra. The algebra we obtain is Morita equivalent to the principal block component of $S_{q}(3,7)$ when $l=3$, and $p=2$. Therefore, $S_{q}(3,8)$ is of wild representation type.

5.4. We consider the algebra $S_{q}(3,4)$ for $l=2$. We will show that it is Morita equivalent to $\mathcal{R}_{4}$, and this is tame (see the proof of [DEMN, 5.7]). By using methods

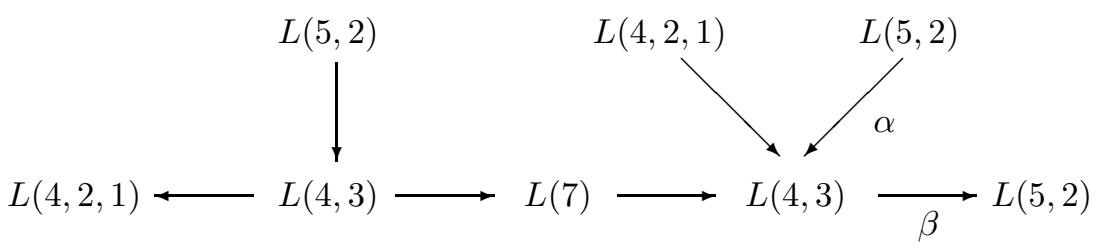

FIGURE 7. 
as before one shows easily that decomposition matrices are

$$
\left(\begin{array}{c}
{[\Delta(4): L(\mu)]} \\
{[\Delta(3,1): L(\mu)]} \\
{\left[\Delta\left(2^{2}\right): L(\mu)\right]} \\
{\left[\Delta\left(2,1^{2}\right): L(\mu)\right]}
\end{array}\right)=\left(\begin{array}{llll}
1 & 1 & 0 & 0 \\
0 & 1 & 1 & 1 \\
0 & 0 & 1 & 1 \\
0 & 0 & 0 & 1
\end{array}\right), \quad\left(\begin{array}{c}
{\left[S^{(4)}: D^{\mu}\right]} \\
{\left[S^{(3,1)}: D^{\mu}\right]} \\
{\left[S^{(2,2)}: D^{\mu}\right]} \\
{\left[S^{\left(2,1^{2}\right)}: D^{\mu}\right]}
\end{array}\right)=\left(\begin{array}{ll}
1 & 0 \\
1 & 1 \\
0 & 1 \\
1 & 1
\end{array}\right) .
$$

Using this and $\S 2.6$ we see that $Y^{(3,1)}$ is uniserial with socle and top $k$ with heart $D^{(3,1)}$. The module $Y^{\left(2^{2}\right)}$ is also uniserial of length three with socle and top $D^{(3,1)}$ with heart $k$. Moreover, $Y^{\left(2,1^{2}\right)}$ is the projective cover of $D^{(3,1)}$ which is uniserial of length five with composition factors $D^{(3,1)}, k, D^{(3,1)}, k, D^{(3,1)}$. (Here we use that $Y^{(2,2)}$ is a quotient of $P(3,1)$.)

The algebra $\operatorname{End}_{\mathcal{H}_{q}(4)}\left(\bigoplus_{\lambda \in \Lambda} Y^{\lambda}\right)$ is the basic algebra for $S_{q}(3,4)$. The Young modules satisfy (essentially) the hypotheses in [Erd2, 3.12] (the composition factor $S_{3}$ in [Erd2, 3.12] is not visible in the endomorphism ring), and hence the basic algebra is isomorphic to the algebra $\mathcal{R}_{4}$.

5.5. Consider now the algebra $S_{q}(3,5)$ for $l=2$. We will show that the principal block component is Morita equivalent to $\mathcal{H}_{4}$ of [Erd2, 3.13], which is tame. The other block is semi-simple and therefore we will have shown that $S_{q}(3,5)$ is tame.

The decomposition matrices for the principal block components are as follows:

$$
\left(\begin{array}{c}
{[\Delta(5): L(\mu)]} \\
{[\Delta(3,2): L(\mu)]} \\
{\left[\Delta\left(3,1^{2}\right): L(\mu)\right]} \\
{\left[\Delta\left(2^{2}, 1\right): L(\mu)\right]}
\end{array}\right)=\left(\begin{array}{llll}
1 & 0 & 1 & 0 \\
0 & 1 & 1 & 1 \\
0 & 0 & 1 & 1 \\
0 & 0 & 0 & 1
\end{array}\right), \quad\left(\begin{array}{c}
{\left[S^{(5)}: D^{\mu}\right]} \\
{\left[S^{(3,2)}: D^{\mu}\right]} \\
{\left[S^{\left(3,1^{2}\right)}: D^{\mu}\right]} \\
{\left[S^{\left(2^{2}, 1\right)}: D^{\mu}\right]}
\end{array}\right)=\left(\begin{array}{ll}
1 & 0 \\
0 & 1 \\
1 & 1 \\
0 & 1
\end{array}\right) .
$$

To prove this, we first find the decomposition matrix for the Weyl modules using techniques that have already been demonstrated in previous examples. From this we see that the Young module $Y^{(3,2)}$ is just $S^{(3,2)}$ and hence $S^{(3,2)}$ is simple. Then the rest of the decomposition matrix for the Hecke algebra follows easily.

The Young module $Y^{\left(3,1^{2}\right)}$ has length four and socle and top each are isomorphic to $k \oplus D^{(3,2)}$. Moreover, $Y^{\left(2^{2}, 1\right)}$ is the projective cover of $D^{(3,2)}$ and it has Loewy length three, the middle is a direct sum $k \oplus D^{(3,2)}$. The endomorphism ring of $\bigoplus_{\lambda} Y^{\lambda}$ is the basic algebra of the principal block of $S_{q}(3,5)$. This algebra is isomorphic to $\mathcal{H}_{4}$, as in [Erd2, 3.13]. Actually, this is proved using the arguments of Erd2 3.14] by letting $E_{1}=k, E_{2}=D^{(3,2)}$ and $E_{3}=Y^{\left(3,1^{2}\right)}$ and $E_{4}=P\left(D^{(3,2)}\right)$. The crucial ingredient is that there is a homomorphism $\pi: E_{4} \rightarrow E_{3}$ and a homomorphism $j: E_{3} \rightarrow E_{4}$ such that the composition $j \pi$ has image $D^{(3,2)} \oplus k$. This gives the commutativity relation.

5.6. We will now prove that the algebra $S_{q}(3,6)$ for $l=2$ is wild. The decomposition matrix for the Weyl modules is given as follows:

$$
\left(\begin{array}{c}
{[\Delta(6): L(\mu)]} \\
{[\Delta(5,1): L(\mu)]} \\
{[\Delta(4,2): L(\mu)]} \\
{\left[\Delta\left(4,1^{2}\right): L(\mu)\right]} \\
{\left[\Delta\left(3^{2}\right): L(\mu)\right]} \\
{\left[\Delta\left(2^{3}\right): L(\mu)\right]}
\end{array}\right)=\left(\begin{array}{cccccc}
1 & 1 & 0(1) & 0 & 0 & 0 \\
0 & 1 & 1 & * & 0 & * \\
0 & 0 & 1 & 1 & 1 & 1(2) \\
0 & 0 & 0 & 1 & 0 & 1 \\
0 & 0 & 0 & 0 & 1 & 1 \\
0 & 0 & 0 & 0 & 0 & 1
\end{array}\right)
$$

Here the composition factors in brackets occur for $p=3$ only. We proceed now by using the argument as in Proposition 5.2(A), for the algebra $S_{q}(3,10)$ when $l=3$. 
We see from the decomposition matrix that the tilting modules $T\left(3^{2}\right)$ and $T\left(4,1^{2}\right)$ are uniserial of length three, with top and socle $L\left(2^{3}\right)$ and the heart $L\left(3^{2}\right)$ and $L\left(4,1^{2}\right)$ respectively. So there is an exact sequence

$$
0 \rightarrow W \rightarrow P\left(2^{3}\right) \rightarrow X \rightarrow 0
$$

and an exact sequence

$$
0 \rightarrow \Delta\left(3^{2}\right) \oplus \Delta\left(4,1^{2}\right) \rightarrow X \rightarrow L\left(2^{3}\right) \rightarrow 0
$$

where $W$ has quotient $\Delta(4,2)$. By the same argument used in Proposition 5.2(A) it follows that the algebra $S_{q}(3,6)$ for $l=2$ is wild.

5.7. Next we show that the algebra $S_{q}(3,7)$ for $l=2$ is wild. The line of reasoning will be similar to the one used to show that $S_{q}(3,11)$ is wild when $l=3$ and $p \neq 2$. The decomposition matrix of the principal block component is of the form

$$
\left(\begin{array}{c}
{[\Delta(7): L(\mu)]} \\
{[\Delta(5,2): L(\mu)]} \\
{\left[\Delta\left(5,1^{2}\right): L(\mu)\right]} \\
{[\Delta(4,2,1): L(\mu)]} \\
{\left[\Delta\left(3^{2}, 1\right): L(\mu)\right]} \\
{\left[\Delta\left(3,2^{2}\right): L(\mu)\right]}
\end{array}\right)=\left(\begin{array}{cccccc}
1 & 0(1) & 1 & 0 & 0 & 0 \\
0 & 1 & 1 & * & * & * \\
0 & 0 & 1 & 1 & 0 & 0 \\
0 & 0 & 0 & 1 & 1 & 1 \\
0 & 0 & 0 & 0 & 1 & 1 \\
0 & 0 & 0 & 0 & 0 & 1
\end{array}\right)
$$

The extra composition factor for $\Delta(7)$ exists only when $p=3$. The submatrix corresponding to the 3-part partitions is the same as for $S_{q}(3,4)$ and actually the corresponding quotient of $S_{q}(3,7)$ is Morita equivalent to $S_{q}(3,4)$, an equivalence is given by tensoring with the $q$-determinant, hence is Morita equivalent to $\mathcal{R}_{4}$.

Assume first that $p \neq 3$, then there is a non-split extension of $L(7)$ with $L\left(5,1^{2}\right)$. Combining this with the information on 3-part partitions we see that the quiver contains

$$
L(7) \longleftrightarrow L\left(5,1^{2}\right) \longleftrightarrow L(4,2,1) \longleftrightarrow L\left(3^{2}, 1\right) \longleftrightarrow L\left(3,2^{2}\right)
$$

There must be arrows joining this quiver with $L(5,2)$ and by the argument as for $S_{q}(3,11)$ and $l=3, p \neq 2$ (Proposition 5.2(B)) we get a wild algebra.

Now assume $p=3$, then the Weyl module $\Delta(7)$ is uniserial, with heart $L(5,2)$ and socle $L\left(5,1^{2}\right)$, so we get a subquiver

$$
(7) \longleftrightarrow(5,2) \longleftrightarrow\left(5,1^{2}\right) \longleftrightarrow(4,2,1) \longleftrightarrow\left(3^{2}, 1\right) \longleftrightarrow\left(3,2^{2}\right) .
$$

Suppose, for a contradiction, this is the full quiver. Then we use the argument as in the proof of $4.3(\mathrm{~B})$ and deduce that the radical of $\Delta(5,2)$ must be uniserial, with composition factors $L\left(5,1^{2}\right), L(4,2,1), L\left(3^{2}, 1\right), L\left(3,2^{2}\right)$, so there is an epimorphism $\pi: P\left(5,1^{2}\right) \rightarrow \operatorname{rad}(\Delta(5,2))$ which must factor through $\Delta\left(5,1^{2}\right)$, a contradiction.

5.8. Finally, we will demonstrate that the algebra $S_{q}(3,8)$ for $l=2$ is wild. If $p=$ 3 , this follows already from $\S 3.4$, so we assume $p \neq 3$. A part of the decomposition 
matrix is given as follows:

$$
\left(\begin{array}{c}
{[\Delta(8): L(\mu)]} \\
{[\Delta(7,1): L(\mu)]} \\
{[\Delta(6,2): L(\mu)]} \\
{[\Delta(5,3): L(\mu)]} \\
{\left[\Delta\left(4^{2}\right): L(\mu)\right]} \\
{\left[\Delta\left(6,1^{2}\right): L(\mu)\right]} \\
{[\Delta(4,3,1): L(\mu)]} \\
{\left[\Delta\left(4,2^{2}\right): L(\mu)\right]} \\
{\left[\Delta\left(3^{2}, 2\right): L(\mu)\right]}
\end{array}\right)=\left(\begin{array}{lllllllll}
1 & 1 & 0 & 0 & 0 & 0 & 0 & 0 & 0 \\
0 & 1 & 1 & 0 & 0 & 1 & 0 & 0 & 0 \\
0 & 0 & 1 & 1 & 0 & * & * & * & * \\
0 & 0 & 0 & 1 & 1 & 0 & * & * & * \\
0 & 0 & 0 & 0 & 1 & 0 & 1 & 0 & 0 \\
0 & 0 & 0 & 0 & 0 & 1 & 0 & 1 & 0 \\
0 & 0 & 0 & 0 & 0 & 0 & 1 & 1 & 1 \\
0 & 0 & 0 & 0 & 0 & 0 & 0 & 1 & 1 \\
0 & 0 & 0 & 0 & 0 & 0 & 0 & 0 & 1
\end{array}\right)
$$

The quotient algebra corresponding to partitions with exactly three parts is Morita equivalent to $S_{q}(3,5)$, that is, to the algebra $\mathcal{H}_{4}$. This gives also part of the quiver. The part corresponding to 2-part partitions is obtained by row- and columnremoval.

We wish to complete the row for $\Delta(7,1)$, that is the last four entries. By the dimensions, these follow if we show that $L\left(6,1^{2}\right)$ must occur. Now $L\left(5,1^{2}\right)$ occurs in $\Delta(7)$, and hence $L\left(6,1^{2}\right) \cong L\left(5,1^{2}\right) \otimes L(1)$ occurs in $\Delta(7) \otimes L(1)$, which has $\Delta$-quotients with weights $(8)$ and $(7,1)$, and the claim follows.

Now we consider the quiver, it contains $L(8) \longleftrightarrow L(7,1)$, and a subquiver of type $\mathcal{H}_{4}$ (Figure 5) with $X_{1}=L\left(6,1^{2}\right), X_{2}=L\left(4,2^{2}\right), X_{3}=L\left(3^{2}, 2\right)$ and $X_{4}=$ $L(4,3,1)$. Moreover, from the decomposition matrix we see that there are arrows $L\left(4^{2}\right) \longleftrightarrow L(4,3,1)$. So we have a subquiver of shape $\mathcal{A}_{2}$ and a subquiver of shape $\mathcal{D}_{5}$. Next consider how $L(6,2)$ is joined. There are three possibilities for the submodule structure of $\Delta(7,1)$, two of them lead to a subquiver which contracts to $\widetilde{\widetilde{D}}_{4}$ and gives a wild algebra. So we are left with the possibility that $\Delta(7,1)$ is uniserial with second socle $L(6,2)$ in which case the shape of the quiver got so far is $E_{8}$. The vertex corresponding to $L(5,3)$ must be joined to at least one of $L\left(4^{2}\right)$ or $L(4,3,1)$ since there is a projective with $\Delta$-quotients $\Delta\left(4^{2}\right)$ and $\Delta(5,3)$ which has a simple top. In both cases the quiver leads to a wild algebra (either $\underset{E_{6}}{\approx}$ or after contraction to $\widetilde{\widetilde{D}}_{4}$ ).

\section{Representation TyPe FOR SOME $S_{q}(4, r)$ AND $S_{q}(5, r)$}

6.1. We first show that $S_{q}(4,7)$ for $p \neq 2$ and $l=3$ is wild. The Weyl module $\Delta(7)$ is uniserial of length three, with heart $L(5,2)$ and socle $L\left(2^{3}, 1\right)$. Hence, the quiver contains

$$
L(7) \longleftrightarrow L(5,2) \longleftrightarrow L\left(2^{3}, 1\right)
$$

The structure of $S_{q}(4,3)$-modules implies that the quiver of $S_{q}(4,7)$ also contains

$$
L\left(4,1^{3}\right) \longleftrightarrow L\left(3,2,1^{2}\right) \longleftrightarrow L\left(2^{3}, 1\right) .
$$

Consider the principal block of $S_{q}(4,4)$ for the same field. There exists a non-split exact sequence

$$
0 \rightarrow L\left(1^{4}\right) \rightarrow \Delta\left(2^{2}\right) \rightarrow L(2,2) \rightarrow 0
$$

Tensoring this exact sequence with $L(1)^{F}$ gives a non-split exact sequence

$$
0 \rightarrow L\left(4,1^{3}\right) \rightarrow \Delta\left(2^{2}\right) \otimes L(1)^{F} \rightarrow L(5,2) \rightarrow 0
$$


Therefore, the quiver also contains $L\left(4,1^{3}\right) \longleftrightarrow L(5,2)$. Combining this information we obtain a subquiver which is an "extended circuit", thus by Proposition 2.3(B), $S_{q}(4,7)$ for $p \neq 2$ and $l=3$ is wild.

6.2. Now we will show that the algebra $S_{q}(4,8)$ for $p \neq 2$ and $l=3$ is wild. The Weyl module $\Delta(8)$ is uniserial of length three with socle $L\left(2^{4}\right)$ and heart $L(5,2,1)$. By tensoring Weyl modules for $S_{q}(4,4)$ with the determinant we see that $\Delta\left(5,1^{3}\right)$ has length two and socle $L\left(3^{2}, 1^{2}\right)$, and $\Delta\left(3^{2}, 1^{2}\right)$ has also length two and socle $L\left(2^{4}\right)$.

Consider $S_{q}(4,5)$ over the same field. There is a non-split exact sequence

$$
0 \rightarrow L\left(2,1^{3}\right) \rightarrow N \rightarrow L\left(2^{2}, 1\right) \rightarrow 0 .
$$

Tensoring this sequence with $L(1)^{F}$ gives a non-split exact sequence with end terms $L\left(5,1^{3}\right)$ and $L(5,2,1)$. Combining this information yields a "circuit" of length four which is joined to another vertex. Hence, by Proposition 2.3(B), $S_{q}(4,8)$ is wild when $p \neq 2$ and $l=3$.

6.3. Consider the algebra $S_{q}(4,4)$ for $l=2$. Here the Weyl module $\Delta(4)$ is uniserial of length three with socle $L\left(1^{4}\right)$ and heart $L(3,1)$. The decomposition matrix for Weyl modules is given by

$$
\left(\begin{array}{c}
{[\Delta(4): L(\mu)]} \\
{[\Delta(3,1): L(\mu)]} \\
{\left[\Delta\left(2^{2}\right): L(\mu)\right.} \\
{\left[\Delta\left(2,1^{2}\right): L(\mu)\right]} \\
{\left[\Delta\left(1^{4}\right): L(\mu)\right]}
\end{array}\right)=\left(\begin{array}{lllll}
1 & 1 & 0 & 0 & 1 \\
0 & 1 & 1 & 1 & 1 \\
0 & 0 & 1 & 1 & 0 \\
0 & 0 & 0 & 1 & 1 \\
0 & 0 & 0 & 0 & 1
\end{array}\right)
$$

The upper left $4 \times 4$ part is obtained from $S_{q}(4,4)$. For the last column, note that $P\left(1^{4}\right)$ is a tilting module and it must be $T(4)$. By considering the decomposition matrix for the Hecke algebra $\mathcal{H}_{q}(4)$ (see 5.4 , add a row $(1,0)$ for $\left.S^{\left(1^{4}\right)}\right)$ we get its $\Delta$-quotients.

From the decomposition matrix and the structure of $\Delta(4)$ one sees that the quiver contains

$$
L(4) \longleftrightarrow L(3,1) \longleftrightarrow L\left(1^{4}\right) \longleftrightarrow L\left(2,1^{2}\right) \longleftrightarrow L\left(2^{2}\right) .
$$

The projective module $P\left(2^{2}\right)$ has a filtration with quotients $\Delta\left(2^{2}\right)$ and $\Delta(3,1)$ (by reciprocity). It has a simple top, hence there must be a non-split extension of $L(3,1)$ with either $L\left(2^{2}\right)$ or with $L\left(2,1^{2}\right)$. In both cases the quiver contains a proper subquiver which is a circuit and by Proposition 2.3(B) the algebra is wild.

6.4. Let $l=2$ and consider $S_{q}(4,5)$. First, the principal block of $S_{q}(4,5)$ has only partitions with three parts, so it is Morita equivalent to the principal block of $S_{q}(3,5)$ and is therefore tame. The other block has only two partitions and is of finite type. Hence, $S_{q}(4,5)$ is of tame representation type for $l=2$.

6.5. Finally, consider the algebra $S_{q}(5,5)$ for $l=2$. We will show that the prinicpal block is wild. First we claim that the decomposition matrix for Weyl modules is as 
follows:

$$
\left(\begin{array}{c}
{[\Delta(5): L(\mu)]} \\
{[\Delta(3,2): L(\mu)]} \\
{\left[\Delta\left(3,1^{2}\right): L(\mu)\right]} \\
{\left[\Delta\left(2^{2}, 1\right): L(\mu)\right]} \\
{\left[\Delta\left(1^{5}\right): L(\mu)\right]}
\end{array}\right)=\left(\begin{array}{lllll}
1 & 0 & 1 & 0 & 1 \\
0 & 1 & 1 & 1 & 0 \\
0 & 0 & 1 & 1 & 1 \\
0 & 0 & 0 & 1 & 0 \\
0 & 0 & 0 & 0 & 1
\end{array}\right)
$$

The upper left $4 \times 4$ part of the decomposition matrix of the Weyl modules is obtained from $S_{q}(3,5)$. It remains to determine the three entries in the last column. Note that $P\left(1^{5}\right)$ is a tilting module and it must be $T(5)$. The last column is now obtained by the argument of 6.3 (using 5.5).

The Weyl module $\Delta(5)$ is uniserial, with middle $L\left(3,1^{2}\right)$ and socle $L\left(1^{5}\right)$. Next observe that $P\left(2^{2}, 1\right)$ is a tilting module and hence is $T(3,2)$. So its socle is simple $L\left(2^{2}, 1\right)$ and this must be the socle of $\Delta(3,2)$. We deduce that $\Delta(3,2)$ is uniserial, with heart $L\left(3,1^{2}\right)$.

Combining these we see that the quiver contains a $\tilde{D}_{4}$-shaped subquiver with center $L\left(3,1^{2}\right)$. Since the quiver must be visible by looking at the second radical layer of Weyl modules, we deduce that $\Delta\left(3,1^{2}\right)$ must have semisimple radical, $L\left(2^{2}, 1\right) \oplus L\left(1^{5}\right)$. Now we obtain as the radical layering of $P\left(2^{2}, 1\right) \cong I\left(2^{2}, 1\right)=$ $T(3,2)$,

$$
\begin{gathered}
L\left(2^{2}, 1\right), \\
L\left(3,1^{2}\right), \\
L(3,2) \oplus L\left(2^{2}, 1\right) \oplus L\left(1^{5}\right), \\
L\left(3,1^{2}\right), \\
L\left(2^{2}, 1\right) .
\end{gathered}
$$

This shows that the algebra $S_{q}(5,5)$ for $l=2$ is wild by comparison with the representations of a quiver of shape $C \widetilde{\widetilde{D}}_{4}$.

\section{REFERENCES}

[Cox1] A.G. Cox, The blocks of the $q$-Schur algebra, J. Algebra 207 (1998), 306-325. MR 99k:16078

[Cox2] A.G. Cox, $\operatorname{Ext}^{1}$ for Weyl modules for $q-G L(2, k)$, Math. Proc. Camb. Phil. Soc. 124 (1998), 231-251. MR 99h:17018

[CE] A.G. Cox, K. Erdmann, On Ext ${ }^{2}$ between Weyl modules for quantum $G L_{n}$, Math. Proc. Camb. Phil. Soc. 128 (2000), 441-463. MR 2000m:20069

[DD] R. Dipper, S. Donkin, Quantum GL $L_{n}$, Proc. London Math. Soc. 63 (1991), 165-211. MR 92g:16055

[DJ1] R. Dipper, G.D. James, Representations of Hecke algebras of general linear groups, Proc. London Math. Soc. 52 (1986), 20-52. MR 88b:20065

[DJ2] R. Dipper, G.D. James, Blocks and idempotents of Hecke algebras of general linear groups, Proc. London Math. Soc. 54 (1987), 57-82. MR 88m:20084

[Don] S. Donkin, The q-Schur algebra, London Mathematical Society Lecture Note Series 253 (1998), CUP. CMP 2000:01

[DEMN] S.R. Doty, K. Erdmann, S. Martin and D.K. Nakano, Representation type of Schur algebras, Math. Zeit. 232 (1999), 137-182. MR 2000k:16011

[DN] S. R. Doty, D. K. Nakano, Semisimplicity of Schur algebras, Math. Proc. Camb. Phil. Soc. 124 (1998), 15-20. MR 99d:16020

[EN] K. Erdmann, D.K. Nakano, Representation type of Hecke algebras of type $A$, to appear in Transactions of AMS.

[Erd1] K. Erdmann, Blocks of tame representation type and related algebras, (Lecture Notes in Math. 1428), Springer-Verlag, New York 1990. MR 91c:20016 
[Erd2] K. Erdmann, Schur algebras of finite type, Quart. J. Math. Oxford (2) 44 (1993), 17-41. MR 93k:16024

[Gr] J. A. Green, Polynomial Representations of $G L_{n}$, (Lecture Notes in Math. 830), Springer-Verlag, New York 1980. MR 83j:20003

[Jam] G.D. James, The decomposition matrices for $G L_{n}(q)$ for $n \leq 10$, Proc. London Math. Soc. 60 (1990), 225-265. MR 91c:20024

[JM] G.D. James, A. Mathas, A $q$-analogue of the Jantzen-Schaper theorem, Proc. London Math. Soc. 74 (1997), 241-274. MR 97j:20013

[Jan] J. C. Jantzen, Representations of Algebraic Groups, Academic Press, Orlando 1987. MR 89c:20001

[Mar] S. Martin, Schur Algebras and Representation Theory, Cambridge Univ. Press, 1993. MR 95f:20071

[PW] B. Parshall, J.P Wang, Quantum linear groups, Memoirs of the AMS 89 (1991).

[Ri1] C.M. Ringel, Tame Algebras, in Representation Theory I, (Lecture Notes in Math. 831), Springer-Verlag (1980), 137-287. MR 82j:16056

[Ri2] C.M. Ringel, The category of modules with good filtration over a quasi-hereditary algebra has almost split sequences, Math. Zeit. 208 (1991), 209-225. MR 93c:16010

[Th] L. Thams, The subcomodule structure of quantum symmetric powers, Bull. Austral. Math. Soc. 50 (1994), 29-39. MR 95f:17016

[U] K. Uno, On representations of non-semisimple specialized Hecke algebras, J. Algebra 149 (1992), 287-312. MR 93h:20044

Mathematical Institute, Oxford University, 24-29 St. Giles, Oxford, OX1 3LB, UK

E-mail address: erdmann@maths.ox.ac.uk

Department of Mathematics, University of Georgia, Athens, Georgia 30602 